\title{
三维非线性多层渗流方程耦合系统的 差分方法*
}

\author{
袁益让 \\ (山东大学数学研究所, 济南 250100)
}

摘要对三维多层非线性渗流方程耦合系统提出适合并行计算的二阶迎风分 数步差分格式, 利用变分形式、能量方法、差分算子乘积交换性、高阶差分算子 的分解、先验估计的理论和技巧, 得到收玫性的最佳阶的 $l^{2}$ 误差估计. 该方法 已成功地应用到油资源运移聚集数值模拟的生产实际中.

\section{关键词 渗流耦合系统 非线性 迎风分数步 收玫性 数值模拟}

\section{1 引言}

在三维多层地下渗流驱动问题的非稳定流计算中，若研究 3 层问题，置于 它们中间的层 (弱渗透层) 仅有垂直流动时, 需要求解下述一类三维多层对流扩 散耦合系统的初边值问题 ${ }^{[1 \sim 4]}$ :

$$
\begin{gathered}
\Phi_{1}(x, y, z) \frac{\partial u}{\partial t}+\boldsymbol{a}(x, y, z, t) \cdot \nabla u \\
-\nabla \cdot\left(K_{1}(x, y, z, u, t) \nabla u\right)+\left.K_{2}(x, y, z, t) \frac{\partial w}{\partial z}\right|_{z=H_{2}} \\
=Q_{1}(x, y, z, t, u), \quad(x, y, z)^{\mathrm{T}} \in \Omega_{1}, \quad t \in J=(0, T], \\
\Phi_{2}(x, y, z) \frac{\partial w}{\partial t}=\frac{\partial}{\partial z}\left(K_{2}(x, y, z, t) \frac{\partial w}{\partial z}\right), \quad(x, y, z)^{\mathrm{T}} \in \Omega_{2}, \quad t \in J,
\end{gathered}
$$

*国家重点基础研究专项经费 (批准号: G19990328)、国家攻关计划、国家自然科学基金 (批准号: 10372052,10271066) 和教育部博士点基金 (批准号: 20030422047) 资助项目

***E-mail: yryuan@sdu.edu.cn 


$$
\begin{aligned}
& \Phi_{3}(x, y, z) \frac{\partial v}{\partial t}+\boldsymbol{b}(x, y, z, t) \cdot \nabla v \\
& -\nabla \cdot\left(K_{3}(x, y, z, v, t) \nabla v\right)-\left.K_{2}(x, y, z, t) \frac{\partial w}{\partial z}\right|_{Z=H_{1}} \\
= & Q_{3}(x, y, z, t, v), \quad(x, y, z)^{\mathrm{T}} \in \Omega_{3}, \quad t \in J,
\end{aligned}
$$

此处

$$
\begin{gathered}
\Omega=\cup_{i=1}^{3} \Omega_{i}, \quad \Omega_{1}=\left\{(x, y) \in \Omega_{0}, H_{2}<z<H_{3}\right\} \\
\Omega_{2}=\left\{(x, y) \in \Omega_{0}, H_{1}<z<H_{2}\right\}, \quad \Omega_{3}=\left\{(x, y) \in \Omega_{0}, 0<z<H_{1}\right\},
\end{gathered}
$$

$\Omega_{0}$ 为图 1 所示的平面有界区域, $\partial \Omega, \partial \Omega_{i}(i=1,2,3)$ 分别为 $\Omega$ 和 $\Omega_{i}$ 的外边界.

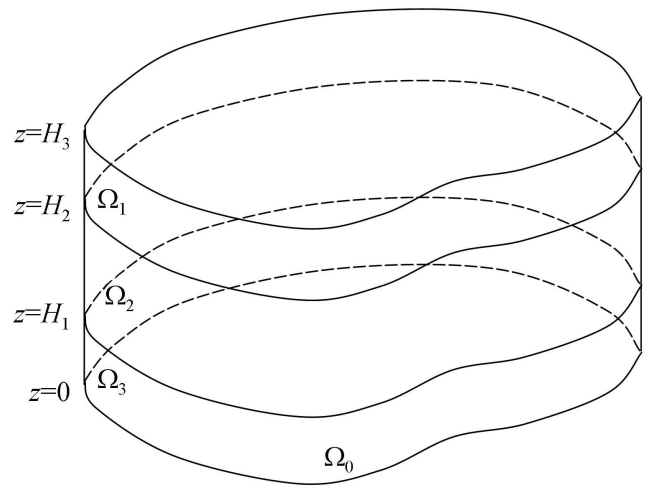

图 1 区域 $\Omega_{1}, \Omega_{2}, \Omega_{3}$ 示意图

初始条件:

$$
\begin{array}{ll}
u(x, y, z, 0)=\Psi_{1}(x, y, z), & (x, y, z)^{\mathrm{T}} \in \Omega_{1}, \\
w(x, y, z, 0)=\Psi_{2}(x, y, z), & (x, y, z)^{\mathrm{T}} \in \Omega_{2}, \\
v(x, y, z, 0)=\Psi_{3}(x, y, z), & (x, y, z)^{\mathrm{T}} \in \Omega_{3} .
\end{array}
$$

边界条件是第 1 型的:

$$
\begin{gathered}
\left.u(x, y, z, t)\right|_{z=H_{3}, \partial \Omega_{1}}=0,\left.\quad w(x, y, z, t)\right|_{\partial \Omega_{2}}=0,\left.\quad v(x, y, z, t)\right|_{z=0, \partial \Omega_{3}}=0, \\
\left.w(x, y, z, t)\right|_{z=H_{2}}=u\left(x, y, H_{2}, t\right), \\
\left.w(x, y, z, t)\right|_{z=H_{1}}=v\left(x, y, H_{1}, t\right), \quad(x, y)^{\mathrm{T}} \in \Omega_{0} \quad \text { (内边界条件). }
\end{gathered}
$$

在渗流力学中, 待求函数 $u, w, v$ 为位势函数, $\nabla u, \nabla v, \frac{\partial w}{\partial z}$ 为 Darcy 速度, $\Phi_{\alpha}(\alpha=1,2,3)$ 为孔隙度函数, $K_{1}(x, y, z, u, t), K_{2}(x, y, z, t)$ 和 $K_{3}(x, y, z, v, t)$ 为 渗透率函数,

$$
\begin{gathered}
\boldsymbol{a}(x, y, z, t)=\left(a_{1}(x, y, z, t), a_{2}(x, y, z, t), a_{3}(x, y, z, t)\right)^{\mathrm{T}} \\
\boldsymbol{b}(x, y, z, t)=\left(b_{1}(x, y, z, t), b_{2}(x, y, z, t), b_{3}(x, y, z, t)\right)^{\mathrm{T}}
\end{gathered}
$$


为相应的对流系数, $Q_{1}(x, y, z, t, u), Q_{3}(x, y, z, t, v)$ 为产量项.

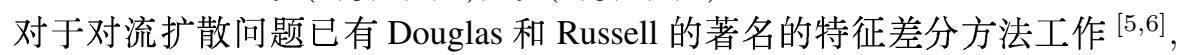
它克服了经典方法可能出现的数值解的振荡和失真 ${ }^{[1,7,8]}$, 解决了用差分方法处 理以对流为主的对流扩散问题. 但特征差分方法有处理边界条件所带来的计算复 杂性 ${ }^{[1,6]}$, Ewing 和 Lazarov 等提出用迎风差分格式来解决这类问题 ${ }^{[9,10]}$. 为解决 大规模科学与工程计算 (节点个数可多达数万乃至数十万个), 需要采用分数步技 术, 将高维问题化为连续解几个一维问题的计算 ${ }^{[7,8,11]}$. 本文从油气资源勘探、 开发和地下水渗流计算的实际问题出发, 研究三维多层非线性地下渗流耦合系 统驱动问题的非稳定渗流计算, 提出适合并行计算的二阶耦合迎风分数步差分 格式，利用变分形式、能量方法、差分算子乘积交换性、高阶差分算子的分解、 先验估计的理论和技巧, 对二阶格式得到收玫性的最佳阶 $l^{2}$ 误差估计. 对于简 化的情形, 即将问题简化为二维平面问题, 且假定问题是线性的, 作者已有初步 成果 ${ }^{[12]}$. 但由于油田勘探的深入发展, 需要寻找 “土豆块闭圈” 中小型油田, 油 田勘探的数值模拟需要向精细化、并行化发展, 需要研究问题的三维和非线性 耦合系统的真实情形. 本文的方法已成功应用到三维油资源运移聚集数值模拟 计算和工程实践中 ${ }^{1,2)}$, 到目前为止还未见这方面成果发表 ${ }^{[10] 3)}$, 我们成功解决 了这一著名的计算数学和石油地质问题 ${ }^{[1,4]}$.

通常问题是正定的, 即满足

$$
\begin{gathered}
0<\Phi_{*} \leqslant \Phi_{a} \leqslant \Phi^{*}, \quad 0<K_{*} \leqslant K_{a} \leqslant K^{*}, \quad a=1,2,3, \\
\left|\frac{\partial K_{1}(u)}{\partial u}\right|+\left|\frac{\partial K_{3}(v)}{\partial v}\right| \leqslant K^{*},
\end{gathered}
$$

此处 $\Phi_{*}, \Phi^{*}, K_{*}, K^{*}$ 均为正常数.

假定问题 (1) (4) 的精确解是正则的, 即

$$
\begin{gathered}
\frac{\partial^{2} u}{\partial t^{2}} \in L^{\infty}\left(L^{\infty}\left(\Omega_{1}\right)\right), \quad u \in L^{\infty}\left(W^{4, \infty}\left(\Omega_{1}\right)\right) \cap W^{1, \infty}\left(W^{1, \infty}\left(\Omega_{1}\right)\right), \\
\frac{\partial^{2} v}{\partial t^{2}} \in L^{\infty}\left(L^{\infty}\left(\Omega_{3}\right)\right), \quad v \in L^{\infty}\left(W^{4, \infty}\left(\Omega_{3}\right)\right) \cap W^{1, \infty}\left(W^{1, \infty}\left(\Omega_{3}\right)\right), \\
\frac{\partial^{2} w}{\partial t^{2}} \in L^{\infty}\left(L^{\infty}\left(\Omega_{2}\right)\right), \quad w \in L^{\infty}\left(W^{4, \infty}\left(\Omega_{2}\right)\right),
\end{gathered}
$$

且 $Q_{1}(x, y, z, t, u)$ 和 $Q_{3}(x, y, z, t, v)$ 在解的 $\varepsilon_{0}$ - 邻域满足 Lipschitz 连续条件, 即存 在常数 $M$, 当 $\left|\varepsilon_{i}\right| \leqslant \varepsilon_{0}(1 \leqslant i \leqslant 4)$ 时, 有

$$
\left|Q_{1}\left(u(x, y, z, t)+\varepsilon_{1}\right)-Q_{1}\left(u(x, y, z, t)+\varepsilon_{2}\right)\right| \leqslant M\left|\varepsilon_{1}-\varepsilon_{2}\right|, \quad(x, y, z, t) \in \Omega_{1} \times J,
$$

1) 山东大学数学研究所, 胜利油田计算中心. 多层油资源运移聚集定量数值模拟技术研究. 1999, 6

2) 山东大学数学研究所, 胜利油田物探研究院. 油资源二次运移聚集并行处理精细模拟技术研究. 2003, 10

3) Ewing R E. Mathematical modelling and simulation for multiphase flow in porous media. An International Workshop on Computation Physics: Fluid Flow and Transport in Porous Media. August 2-6, 1999, Beijing 


$$
\left|Q_{3}\left(v(x, y, z, t)+\varepsilon_{3}\right)-Q_{3}\left(v(x, y, z, t)+\varepsilon_{4}\right)\right| \leqslant M\left|\varepsilon_{3}-\varepsilon_{4}\right|, \quad(x, y, z, t) \in \Omega_{3} \times J .
$$

本文中记号 $M$ 和 $\varepsilon$ 分别表示普通的正常数和小的正数，在不同处可有不同 的含义.

\section{2 二阶迎风分数步差分格式}

为了用差分方法求解, 我们用网格区域 $\Omega_{0, h}$ 代替 $\Omega_{0}$ (见图 2), 在平面 $(x, y)$ 上步长为 $h_{1}, x_{i}=i h_{1}, y_{j}=j h_{1}$,

$$
\Omega_{0, h}=\left\{\left(x_{i}, y_{j}\right) \mid i_{1}(j)<i<i_{2}(j), j_{1}(i)<j<j_{2}(i)\right\} .
$$

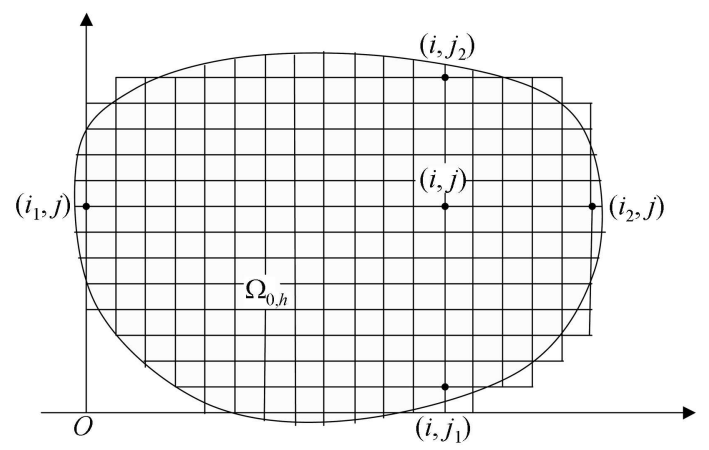

图 2 网域 $\Omega_{0, h}$ 示意图

在 $z$ 方向步长为 $h_{2}, z_{k}=H_{2}+k h_{2}, h_{2}=\left(H_{3}-H_{2}\right) / N_{1}$, 此处 $N_{1}$ 为某一正 整数. $t^{n}=n \Delta t$, 用 $\Omega_{1, h}$ 代替 $\Omega_{1}$,

$$
\Omega_{1, h}=\left\{\left(x_{i}, y_{j}, z_{k}\right) \mid i_{1}(j)<i<i_{2}(j), j_{1}(i)<j<j_{2}(i), 0<k<N_{1}\right\} .
$$

用 $\partial \Omega_{1, h}$ 和 $\partial \Omega_{0, h}$ 分别表示 $\Omega_{1, h}$ 和 $\Omega_{0, h}$ 的边界. 类似地, 对 $\Omega_{2}$ 在 $z$ 方向的 步长取为 $h_{3}, z_{k}=H_{1}+k h_{3}, h_{3}=\left(H_{2}-H_{1}\right) / N_{2}$, 用 $\Omega_{2, h}$ 代替 $\Omega_{2}$,

$$
\Omega_{2, h}=\left\{\left(x_{i}, y_{j}, z_{k}\right) \mid i_{1}(j)<i<i_{2}(j), j_{1}(i)<j<j_{2}(i), 0<k<N_{2}\right\},
$$

对 $\Omega_{3}$ 在 $z$ 方向的步长取为 $h_{4}, z_{k}=k h_{4}, h_{4}=H_{1} / N_{3}$, 同样用 $\Omega_{3, h}$ 代替 $\Omega_{3}$,

$$
\Omega_{3, h}=\left\{\left(x_{i}, y_{j}, z_{k}\right) \mid i_{1}(j)<i<i_{2}(j), j_{1}(i)<j<j_{2}(i), 0<k<N_{3}\right\},
$$

此处 $N_{2}$ 和 $N_{3}$ 为正整数, $\partial \Omega_{2, h}$ 和 $\partial \Omega_{3, h}$ 表示 $\Omega_{2, h}$ 和 $\Omega_{3, h}$ 的外侧边界. 记

$$
U\left(x_{i}, y_{j}, z_{k}, t^{n}\right)=: U_{i j k}^{n}, \quad V\left(x_{i}, y_{j}, z_{k}, t^{n}\right)=: V_{i j k}^{n}, \quad W\left(x_{i}, y_{j}, z_{k}, t^{n}\right)=: W_{i j k}^{n} .
$$

记 $\delta_{x}, \delta_{y}, \delta_{z}, \delta_{\vec{x}}, \delta_{\vec{y}}, \delta_{\vec{z}}$ 分别为 $x, y$ 和 $z$ 方向的向前、向后差商算子, $d_{t} U^{n}$ 为网 格函数 $U_{i j k}^{n}$ 在 $t=t_{n}$ 的向前差商.

为了得到高精度计算格式，在 $\Omega_{2}$ 上对方程 (1a) 在 $\left(x, y, z_{N_{2}-\frac{1}{2}}, t\right)$ 点展开:

$$
\begin{aligned}
& {\left[K_{2}(x, y, z, t) \frac{\partial w}{\partial z}\right]_{N_{2}-\frac{1}{2}} } \\
= & {\left[K_{2}(x, y, z, t) \frac{\partial w}{\partial z}\right]_{N_{2}}-\frac{h_{3}}{2}\left[\frac{\partial}{\partial z}\left(K_{2}(x, y, z, t) \frac{\partial w}{\partial z}\right)\right]_{N_{2}}+O\left(h_{3}^{2}\right), }
\end{aligned}
$$


于是得

$$
\left[K_{2}(x, y, z, t) \frac{\partial w}{\partial z}\right]_{N_{2}}=\left[K_{2}(x, y, z, t) \frac{\partial w}{\partial z}\right]_{N_{2}-\frac{1}{2}}+\frac{h_{3}}{2}\left[\Phi_{2}(x, y, z) \frac{\partial w}{\partial t}\right]_{N_{2}}+O\left(h_{3}^{2}\right) .
$$

类似地由方程 $(1 \mathrm{c})$ 可得

$$
\left[K_{2}(x, y, z, t) \frac{\partial w}{\partial z}\right]_{0}=\left[K_{2}(x, y, z, t) \frac{\partial w}{\partial z}\right]_{1 / 2}-\frac{h_{3}}{2}\left[\Phi_{2}(x, y, z) \frac{\partial w}{\partial t}\right]_{0}+O\left(h_{3}^{2}\right) .
$$

于是在 $\Omega_{1}$ 上点 $\left(x, y, H_{2}, t\right)$ 处有

$$
\begin{aligned}
& \Phi_{1}(x, y, z) \frac{\partial u}{\partial t}+\boldsymbol{a}(x, y, z, t) \cdot \nabla u-\nabla \cdot\left(K_{1}(x, y, z, u, t) \nabla u\right) \\
& +\frac{h_{3}}{2}\left[\Phi_{2}\left(x, y, H_{2}\right) \frac{\partial u}{\partial t}\right]+\left[K_{2}(x, y, z, t) \frac{\partial w}{\partial z}\right]_{N_{2}-\frac{1}{2}}+O\left(h_{3}^{2}\right) \\
= & Q_{1}(u),
\end{aligned}
$$

即

$$
\begin{aligned}
& \hat{\Phi}_{1}\left(x, y, z, H_{2}\right) \frac{\partial u}{\partial t}+\boldsymbol{a}(x, y, z, t) \cdot \nabla u-\nabla \cdot\left(K_{1}(x, y, z, u, t) \nabla u\right) \\
= & -\left[K_{2}(x, y, z, t) \frac{\partial w}{\partial z}\right]_{N_{2}-\frac{1}{2}}+Q_{1}(u)+O\left(h_{3}^{2}\right),
\end{aligned}
$$

此处

$$
\hat{\Phi}_{1}\left(x, y, z, H_{2}\right)=\Phi_{1}(x, y, z)+\frac{h_{3}}{2} \Phi_{2}\left(x, y, H_{2}\right) .
$$

类似地在 $\Omega_{3}$ 上点 $\left(x, y, H_{1}, t\right)$ 处有

$$
\begin{aligned}
& \hat{\Phi}_{3}\left(x, y, z, H_{1}\right) \frac{\partial v}{\partial t}+\boldsymbol{b}(x, y, z, t) \cdot \nabla v-\nabla \cdot\left(K_{3}(x, y, z, v, t) \nabla v\right) \\
= & {\left[K_{2}(x, y, z, t) \frac{\partial w}{\partial z}\right]_{1 / 2}+Q_{3}(v)+O\left(h_{3}^{2}\right), }
\end{aligned}
$$

此处

$$
\hat{\Phi}_{3}\left(x, y, z, H_{1}\right)=\Phi_{3}(x, y, z)+\frac{h_{3}}{2} \Phi_{2}\left(x, y, H_{1}\right) .
$$

记

$$
\begin{aligned}
K\left(U^{n}\right)_{i+\frac{1}{2}, j k} & :=\left[K\left(x_{i}, y_{j}, z_{k}, U_{i j k}^{n}\right)+K\left(x_{i+1}, y_{j}, z_{k}, U_{i+1, j k}^{n}\right)\right] / 2, \\
K\left(U^{n}\right)_{i, j+\frac{1}{2}, k} & :=\left[K\left(x_{i}, y_{j}, z_{k}, U_{i j k}^{n}\right)+K\left(x_{i}, y_{j+1}, z_{k}, U_{i, j+1, j}^{n}\right)\right] / 2, \\
K\left(U^{n}\right)_{i j, k+\frac{1}{2}} & :=\left[K\left(x_{i}, y_{j}, z_{k}, U_{i j k}^{n}\right)+K\left(x_{i}, y_{j}, z_{k+1}, U_{i j, k+1}^{n}\right)\right] / 2 .
\end{aligned}
$$

设

$$
\begin{aligned}
& \delta_{x}\left(K\left(U^{n}\right) \delta_{\bar{x}} u^{n+1}\right)_{i j k} \\
= & h_{1}^{-2}\left[K\left(U^{n}\right)_{i+\frac{1}{2}, j k}\left(u_{i+1, j k}^{n+1}-u_{i j k}^{n+1}\right)-K\left(U^{n}\right)_{i-\frac{1}{2}, j k}\left(u_{i j k}^{n+1}-u_{i-1, j k}^{n+1}\right)\right], \\
& \delta_{y}\left(K\left(U^{n}\right) \delta_{\bar{y}} u^{n+1}\right)_{i j k} \\
= & h_{1}^{-2}\left[K\left(U^{n}\right)_{i, j+\frac{1}{2}, k}\left(u_{i, j+1, k}^{n+1}-u_{i j k}^{n+1}\right)-K\left(U^{n}\right)_{i, j-\frac{1}{2}, k}\left(u_{i j k}^{n+1}-u_{i, j-1, k}^{n+1}\right)\right],
\end{aligned}
$$




$$
\begin{aligned}
& \delta_{z}\left(K\left(U^{n}\right) \delta_{\bar{z}} u^{n+1}\right)_{i j k} \\
= & h_{2}^{-2}\left[K\left(U^{n}\right)_{i j, k+\frac{1}{2}}\left(u_{i j, k+1}^{n+1}-u_{i j k}^{n+1}\right)-K\left(U^{n}\right)_{i j, k-\frac{1}{2}}\left(u_{i j k}^{n+1}-u_{i j, k-1}^{n+1}\right)\right], \\
& \nabla_{h}\left(K\left(U^{n}\right) \nabla_{h} u^{n+1}\right)_{i j k} \\
= & \delta_{x}\left(K\left(U^{n}\right) \delta_{\bar{x}} u^{n+1}\right)_{i j k}+\delta_{y}\left(K\left(U^{n}\right) \delta_{\bar{y}} u^{n+1}\right)_{i j k}+\delta_{z}\left(K\left(U^{n}\right) \delta_{\bar{z}} u^{n+1}\right)_{i j k} .
\end{aligned}
$$

方程 (5a) 可近似分裂为

$$
\begin{aligned}
& \left(1-\frac{\Delta t}{\hat{\Phi}_{1}} \frac{\partial}{\partial x}\left(K_{1} \frac{\partial}{\partial x}\right)+\frac{\Delta t}{\hat{\Phi}_{1}} a_{1} \frac{\partial}{\partial x}\right)\left(1-\frac{\Delta t}{\hat{\Phi}_{1}} \frac{\partial}{\partial y}\left(K_{1} \frac{\partial}{\partial y}\right)+\frac{\Delta t}{\hat{\Phi}_{1}} a_{2} \frac{\partial}{\partial y}\right) \\
& \times\left(1-\frac{\Delta t}{\hat{\Phi}_{1}} \frac{\partial}{\partial z}\left(K_{1} \frac{\partial}{\partial z}\right)+\frac{\Delta t}{\hat{\Phi}_{1}} a_{3} \frac{\partial}{\partial z}\right) u^{n+1} \\
= & u^{n}-\frac{\Delta t}{\hat{\Phi}_{1}}\left\{\left(K_{2} \frac{\partial w^{n+1}}{\partial z}\right)_{N_{2}-\frac{1}{2}}-Q_{1}\left(x, y, z, t^{n+1}, u^{n+1}\right)\right\},
\end{aligned}
$$

其对应的二阶迎风数步差分格式为

$$
\begin{aligned}
&\left(\hat{\Phi}_{1}-\Delta t\left(1+\frac{h_{1}}{2} \frac{\left|a_{1}^{n}\right|}{K_{1}\left(U^{n}\right)}\right)^{-1} \delta_{x}\left(K_{1}\left(U^{n}\right) \delta_{\bar{x}}\right)+\Delta t \delta_{a_{1}^{n}, U^{n}, x}\right) U_{i j k}^{n+\frac{1}{3}} \\
&= \hat{\Phi}_{1, i j k} U_{i j k}^{n}+\Delta t\left\{-K_{2, i j}^{n}, N_{2}-\frac{1}{2} \delta_{\bar{z}} W_{i j, N_{2}}^{n+1}+Q_{1}\left(x_{i}, y_{j}, z_{k}, U_{i j k}^{n+1}\right)\right\}, \\
& i_{1}(j)<i<i_{2}(j), \\
& U_{i j k}^{n+\frac{1}{3}}=0, \quad\left(x_{i}, y_{j}, z_{k}\right)^{\mathrm{T}} \in \partial \Omega_{1, h}, \\
&\left(\hat{\Phi}_{1}-\Delta t\left(1+\frac{h_{1}}{2} \frac{\left|a_{2}^{n}\right|}{K_{1}\left(U^{n}\right)}\right)^{-1} \delta_{y}\left(K_{1}\left(U^{n}\right) \delta_{\bar{y}}\right)+\Delta t \delta_{a_{2}^{n}, U^{n}, y}\right) U_{i j k}^{n+\frac{2}{3}} \\
&= \hat{\Phi}_{1, i j k} U_{i j k}^{n+\frac{1}{3}}, \quad j_{1}(i)<j<j_{2}(i), \\
& U_{i j k}^{n+\frac{2}{3}}=0, \quad\left(x_{i}, y_{j}, z_{k}\right)^{\mathrm{T}} \in \partial \Omega_{1, h}, \\
&\left(\hat{\Phi}_{1}-\Delta t\left(1+\frac{h_{1}}{2} \frac{\left|a_{3}^{n}\right|}{K_{1}\left(U^{n}\right)}\right)^{-1} \delta_{z}\left(K_{1}\left(U^{n}\right) \delta_{\bar{z}}\right)+\Delta t \delta_{a_{3}^{n}, U^{n}, z}\right) U_{i j k}^{n+1} \\
&= \hat{\Phi}_{1, i j k} U_{i j k}^{n+\frac{2}{3}}, \quad 0<k<N_{1}, \\
& U_{i j k}^{n+1}=0, \quad\left(x_{i}, y_{j}, z_{k}\right)^{\mathrm{T}} \in \partial \Omega_{1, h},
\end{aligned}
$$

此处

$$
\begin{aligned}
\delta_{a_{1}^{n}, U^{n}, x} u_{i j k}= & a_{1, i j k}^{n}\left[H\left(a_{1, i j k}^{n}\right) K_{1}\left(U^{n}\right)_{i j k}^{-1} \cdot K_{1}\left(U^{n}\right)_{i-\frac{1}{2}, j k} \delta_{\bar{x}}\right. \\
& \left.+\left(1-H\left(a_{1, i j k}^{n}\right)\right) K_{1}\left(U^{n}\right)_{i j k}^{-1} \cdot K_{1}\left(U^{n}\right)_{i+\frac{1}{2}, j k} \delta_{x}\right] u_{i j k}, \\
\delta_{a_{2}^{n}, U^{n}, y} u_{i j k}= & a_{2, i j k}^{n}\left[H\left(a_{2, i j k}^{n}\right) K_{1}\left(U^{n}\right)_{i j k}^{-1} \cdot K_{1}\left(U^{n}\right)_{i, j-\frac{1}{2}, k} \delta_{\bar{y}}\right. \\
& \left.+\left(1-H\left(a_{2, i j k}^{n}\right)\right) K_{1}\left(U^{n}\right)_{i j k}^{-1} \cdot K_{1}\left(U^{n}\right)_{i, j+\frac{1}{2}, k} \delta_{y}\right] u_{i j k}, \\
\delta_{a_{3}^{n}, U^{n}, z} u_{i j k}= & a_{3, i j k}^{n}\left[H\left(a_{3, i j k}^{n}\right) K_{1}\left(U^{n}\right)_{i j k}^{-1} \cdot K_{1}\left(U^{n}\right)_{i j, k-\frac{1}{2}} \delta_{\bar{z}}\right. \\
& \left.+\left(1-H\left(a_{3, i j k}^{n}\right)\right) K_{1}\left(U^{n}\right)_{i j k}^{-1} \cdot K_{1}\left(U^{n}\right)_{i j, k+\frac{1}{2}} \delta_{z}\right] u_{i j k},
\end{aligned}
$$




$$
H(z)=\left\{\begin{array}{cc}
1, & z \geqslant 0 \\
0, & z<0
\end{array}\right.
$$

在实际计算时, (7a) 式中 $\delta_{\bar{z}} W_{i j, N_{2}}^{n+1}$ 近似地取为 $\delta_{\bar{z}} W_{i j, N_{2}}^{n}, U_{i j k}^{n+1}$ 近似地取为 $U_{i j k}^{n}$. 方程 $(1 b)$ 的差分格式是

$$
\Phi_{2, i j k} \frac{W_{i j k}^{n+1}-W_{i j k}^{n}}{\Delta t}=\delta_{z}\left(K_{2}^{n} \delta_{\bar{z}} W^{n+1}\right)_{i j k}, \quad 0<k<N_{2}, \quad(i, j) \in \Omega_{0, h} .
$$

方程 (1c) 可近似分裂为

$$
\begin{aligned}
& \left(1-\frac{\Delta t}{\hat{\Phi}_{3}} \frac{\partial}{\partial x}\left(K_{3} \frac{\partial}{\partial x}\right)+\frac{\Delta t}{\hat{\Phi}_{3}} b_{1} \frac{\partial}{\partial x}\right)\left(1-\frac{\Delta t}{\hat{\Phi}_{3}} \frac{\partial}{\partial y}\left(K_{3} \frac{\partial}{\partial y}\right)+\frac{\Delta t}{\hat{\Phi}_{3}} b_{2} \frac{\partial}{\partial y}\right) \\
& \times\left(1-\frac{\Delta t}{\hat{\Phi}_{3}} \frac{\partial}{\partial z}\left(K_{3} \frac{\partial}{\partial z}\right)+\frac{\Delta t}{\hat{\Phi}_{3}} b_{3} \frac{\partial}{\partial z}\right) v_{i j k}^{n+1} \\
= & v_{i j k}^{n+1}+\frac{\Delta t}{\hat{\Phi}_{3}}\left\{\left(K_{2} \frac{\partial w^{n+1}}{\partial z}\right)_{1 / 2}+Q_{3}\left(x_{i}, y_{j}, z_{k}, t^{n+1}, v_{i j k}^{n+1}\right)\right\},
\end{aligned}
$$

其对应的迎风分数步差分格式为

$$
\begin{aligned}
&\left(\hat{\Phi}_{3}-\Delta t\left(1+\frac{h_{1}}{2} \frac{\left|b_{1}^{n}\right|}{K_{3}\left(V^{n}\right)}\right)^{-1} \delta_{x}\left(K_{3}\left(V^{n}\right) \delta_{\bar{x}}\right)+\Delta t \delta_{b_{1}^{n}, V^{n}, x}\right) V_{i j k}^{n+\frac{1}{3}} \\
&= \hat{\Phi}_{3, i j k} V_{i j k}^{n}+\Delta t\left\{K_{2, i j, 1 / 2}^{n} \delta_{z} W_{i j, 0}^{n+1}+Q_{3}\left(x_{i}, y_{j}, z_{k}, t^{n}, V_{i j k}^{n+1}\right)\right\}, \\
& i_{1}(j)<i<i_{2}(j), \\
& V_{i j k}^{n+\frac{1}{3}}=0, \quad\left(x_{i}, y_{j}, z_{k}\right)^{\mathrm{T}} \in \partial \Omega_{3, h}, \\
&\left(\hat{\Phi}_{3}-\Delta t\left(1+\frac{h_{1}}{2} \frac{\left|b_{2}^{n}\right|}{K_{3}\left(V^{n}\right)}\right)^{-1} \delta_{y}\left(K_{3}\left(V^{n}\right) \delta_{\bar{y}}\right)+\Delta t \delta_{b_{2}^{n}, V^{n}, y}\right) V_{i j k}^{n+\frac{2}{3}} \\
&= \hat{\Phi}_{3, i j k} V_{i j k}^{n+\frac{1}{3}}, \quad j_{1}(i)<j<j_{2}(i), \\
& V_{i j k}^{n+\frac{2}{3}}=0, \quad\left(x_{i}, y_{j}, z_{k}\right)^{\mathrm{T}} \in \partial \Omega_{3, h}, \\
&\left(\hat{\Phi}_{3}-\Delta t\left(1+\frac{h_{1}}{2} \frac{b_{3}^{n}}{K_{3}\left(V^{n}\right)}\right)^{-1} \delta_{z}\left(K_{3}\left(V^{n}\right) \delta_{\bar{z}}+\Delta t \delta_{b_{3}^{n}, V^{n}, z}\right) V_{i j k}^{n+1}\right. \\
&= \hat{\Phi}_{3, i j k} V_{i j k}^{n+\frac{2}{3}}, \quad 0<k<N_{3}, \\
& V_{i j k}^{n+1}=0, \quad\left(x_{i}, y_{j}, z_{h}\right)^{\mathrm{T}} \in \partial \Omega_{3 h},
\end{aligned}
$$

此处算子 $\delta_{b_{1}^{n}, V^{n}, x}, \delta_{b_{2}^{n}, V^{n}, y}, \delta_{b_{3}^{n}, V^{n}, z}$ 的定义和 $\delta_{a_{1}^{n}, V^{n}, x}, \delta_{a_{2}^{n}, V^{n}, y}, \delta_{a_{3}^{n}, V^{n}, z}$ 是一样 的. 在实际计算时, (10a) 式中 $\delta_{z} w_{i j, 0}^{n+1}$ 近似地取为 $\delta_{z} w_{i j, 0}^{n}, V_{i j k}^{n+1}$ 近似地取为 $V_{i j k}^{n}$. 差分格式 (7)、(8) 和 (10) 式的计算程序是: 已知时刻 $t=t^{n}$ 的差分解为 $\left\{U_{i j k}^{n}\right.$, $\left.W_{i j k}^{n}, V_{i j k}^{n}\right\}$ 时, 寻求下一时刻 $t=t^{n+1}$ 的 $\left\{U_{i j k}^{n+1}, W_{i j k}^{n+1}, V_{i j k}^{n+1}\right\}$. 首先由 (7a) 和 (7b) 式用追赶法求出过渡层的解 $\left\{U_{i j k}^{n+\frac{1}{3}}\right\}$, 再由 (7c) 和 (7d) 式求出 $\left\{U_{i j k}^{n+\frac{2}{3}}\right\}$, 以后再由 (7e) 和 (7f) 式求出 $\left\{U_{i j k}^{n+1}\right\}$. 与此同时可并行地由 (10a) 和 (10b) 式用追赶法求出过 渡层的解 $\left\{V_{i j k}^{n+\frac{1}{3}}\right\}$, 再由 (10c) 和 (10d) 式求出 $\left\{V_{i j k}^{n+\frac{2}{3}}\right\}$, 以后再由 (10e) 和 (10f) 式 
求出 $\left\{V_{i j k}^{n+1}\right\}$. 最后由 (8) 式利用内边界条件 (3b) 求出 $\left\{W_{i j k}^{n+1}\right\}$. 由正定性条件 (4), 此差分解存在且唯一.

\section{3 收敛性分析}

为理论分析简便，设

$$
\begin{gathered}
\Omega=\{(x, y, z) \mid 0<x<1,0<y<1,0<z<3\}, \\
\left.\Omega_{0}=\{(x, y) \mid 0<x<1,0<y<1)\right\}, \\
\Omega_{1}=\{(x, y, z) \mid 0<x<1,0<y<1,2<z<3\}, \\
\Omega_{2}=\{(x, y, z) \mid 0<x<1,0<y<1,1<z<2\}, \\
\Omega_{3}=\{(x, y, z) \mid 0<x<1,0<y<1,0<z<1\},
\end{gathered}
$$

$h=1 / N, t^{n}=n \Delta t$. 定义网络函数空间 $H_{h}$ 的内积 ${ }^{[13 \sim 15]}$, 对三维网格区域, $\forall \omega, x \in H_{h}$,

$$
\begin{aligned}
\langle\omega, x\rangle_{\Omega_{i}}=\langle\omega, x\rangle_{i} & =\sum_{i, j, k=1}^{N-1} \omega_{i j k} x_{i j k} h^{3}, \\
\langle\omega, x\rangle_{\Omega_{i, 1}}=\langle\omega, x\rangle_{i, 1} & =\sum_{j, k=1}^{N-1} \sum_{i=1}^{N} \omega_{i j k} x_{i j k} h^{3}, \\
\langle\omega, x\rangle_{\Omega_{i, 2}}=\langle\omega, x\rangle_{i, 2} & =\sum_{i, k=1}^{N-1} \sum_{j=1}^{N} \omega_{i j k} x_{i j k} h^{3}, \\
\langle\omega, x\rangle_{\Omega_{i, 3}}=\langle\omega, x\rangle_{i, 3} & =\sum_{i, j=1}^{N-1} \sum_{k=1}^{N} \omega_{i j k} x_{i j k} h^{3},
\end{aligned}
$$

其相应的范数为

$$
\begin{aligned}
\left\|\omega^{n}\right\|_{\Omega_{i}}=\left\|\omega^{n}\right\|_{i} & =\left(\sum_{i, j, k=1}^{N-1}\left(\omega_{i j k}^{n}\right)^{2} h^{3}\right)^{1 / 2} \\
\left\|\delta_{\bar{x}} \omega^{n}\right\|_{\Omega_{i}}=\left\|\delta_{\bar{x}} \omega^{n}\right\|_{i} & =\left(\sum_{j, k=1}^{N-1} \sum_{i=1}^{N}\left(\delta_{\bar{x}} \omega_{i j k}^{n}\right)^{2} h^{3}\right)^{1 / 2}, \\
\left\|\delta_{\bar{y}} \omega^{n}\right\|_{\Omega_{i}}=\left\|\delta_{\bar{y}} \omega^{n}\right\|_{i} & =\left(\sum_{i, k=1}^{N-1} \sum_{j=1}^{N}\left(\delta_{\bar{y}} \omega_{i j k}^{n}\right)^{2} h^{3}\right)^{1 / 2}, \\
\left\|\delta_{\bar{z}} \omega^{n}\right\|_{\Omega_{i}}=\left\|\delta_{\bar{z}} \omega^{n}\right\|_{i} & =\left(\sum_{i, j=1}^{N-1} \sum_{k=1}^{N}\left(\delta_{\bar{z}} \omega_{i j k}^{n}\right)^{2} h^{3}\right)^{1 / 2} .
\end{aligned}
$$

在通常情形, 为了简便, 将内积 $\langle\cdot, \cdot\rangle_{i}$ 和范数 $\|\cdot\|$ 的下标省略. 
首先对格式 (7)、(8) 和 (10) 式进行收敛性分析. 设 $u, v, w$ 为问题 (1) (4) 的 精确解, $U, V, W$ 为格式 (7)、(8) 和 (10) 式的差分解, 记误差函数为

$$
\zeta=u-U, \quad \zeta=v-V, \quad \omega=w-W .
$$

方程 $(7 \mathrm{a}) \sim(7 \mathrm{f})$ 消去 $U^{n+\frac{1}{3}}, U^{n+\frac{2}{3}}$ 可得下述等价的差分方程:

$$
\begin{aligned}
& \hat{\Phi}_{1, i j k} \frac{U_{i j k}^{n+1}-U_{i j k}^{n}}{\Delta t}-\left\{\left(1+\frac{h}{2} \frac{\left|a_{1, i j k}^{n}\right|}{K_{1}\left(U^{n}\right)_{i j k}}\right)^{-1} \delta_{x}\left(K_{1}\left(U^{n}\right) \delta_{\bar{x}}\right)\right. \\
& +\left(1+\frac{h}{2} \frac{\left|a_{2, i j k}^{n}\right|}{K_{1}\left(U^{n}\right)_{i j k}}\right)^{-1} \delta_{y}\left(K_{1}\left(U^{n}\right) \delta_{\bar{y}}\right) \\
& \left.+\left(1+\frac{h}{2} \frac{\left|a_{3, i j k}^{n}\right|}{K\left(U^{n}\right)_{i j k}}\right)^{-1} \delta_{z}\left(K_{1}\left|U^{n}\right|\right) \delta_{\bar{z}}\right\} U_{i j k}^{n+1} \\
& +\delta_{a_{1}^{n}, U^{n}, x} U_{i j k}^{n+1}+\delta_{a_{2}^{n}, U^{n}, y} U_{i j k}^{n+1}+\delta_{a_{3}^{n}, U^{n}, z} U_{i j k}^{n+1} \\
& +\Delta t\left\{( 1 + \frac { h } { 2 } \frac { | a _ { 1 , i j k } ^ { n } | } { K _ { 1 } ( U ^ { n } ) _ { i j k } } ) ^ { - 1 } \delta _ { x } \left(K _ { 1 } ( U ^ { n } ) \delta _ { \overline { x } } \left(\hat{\Phi}_{1}^{-1}\left(1+\frac{h}{2} \frac{\left|a_{2}^{n}\right|}{K_{1}\left(U^{n}\right)_{i j k}}\right)^{-1}\right.\right.\right. \\
& \left.\times \delta_{y}\left(K_{1}\left(U^{n}\right) \delta_{\bar{y}} U^{n+1}\right) \cdot\right)_{i j k}+\left(1+\frac{h}{2} \frac{\left|a_{1, i j k}^{n}\right|}{K_{1}\left(U^{n}\right)_{i j k}}\right)^{-1} \delta_{x}\left(K_{1}\left(U^{n}\right)\right. \\
& \times \delta_{\bar{x}}\left(\hat{\Phi}_{1}^{-1}\left(1+\frac{h}{2} \frac{\left|a_{3}^{n}\right|}{K_{1}\left(U^{n}\right)}\right)^{-1} \delta_{z}\left(K_{1}\left(U^{n}\right) \delta_{\bar{z}} U^{n+1}\right) \cdot\right)_{i j k} \\
& +\left(1+\frac{h}{2} \frac{\left|a_{2, i j k}^{n}\right|}{K_{1}\left(U^{n}\right)_{i j k}}\right)^{-1} \delta_{y}\left(K _ { 1 } ( U ^ { n } ) \delta _ { \overline { y } } \left(\hat{\Phi}_{1}^{-1}\left(1+\frac{h}{2} \frac{a_{3}^{n}}{K_{1}\left(U^{n}\right)}\right)^{-1}\right.\right. \\
& \left.\left.\times \delta_{z}\left(K_{1}\left(U^{n}\right) \delta_{\bar{z}} U^{n+1}\right) \cdot\right)_{i j k}\right\}-\Delta t\left\{( 1 + \frac { h } { 2 } \frac { a _ { 1 , i j k } ^ { n } } { K _ { 1 } ( U ^ { n } ) _ { i j k } } ) ^ { - 1 } \delta _ { x } \left(K_{1}\left(U^{n}\right)\right.\right. \\
& \left.\times \delta_{\bar{x}}\left(\hat{\Phi}_{1}^{-1} \delta_{a_{2}^{n}, U^{n}, y} U^{n+1}\right)\right)_{i j k}\left(1+\frac{h}{2} \frac{\left|a_{1, i j k}^{n}\right|}{K_{1}\left(U^{n}\right)_{i j k}}\right)^{-1} \delta_{x}\left(K_{1}\left(U^{n}\right)\right. \\
& \left.\times \delta_{\bar{x}}\left(\hat{\Phi}_{1}^{-1} \delta_{a_{3}^{n}, U^{n}, z} U^{n+1}\right)\right)_{i j k} \\
& +\delta_{a_{1}^{n}, U^{n}, x}\left(\hat{\Phi}_{1}^{-1}\left(1+\frac{h}{2} \frac{\left|a_{2}^{n}\right|}{K_{1}\left(U^{n}\right)}\right)^{-1} \delta_{y}\left(K_{1}\left(U^{n}\right) \delta_{\bar{y}} U^{n+1}\right)\right)_{i j k} \\
& +\delta_{a_{1}^{n}, U^{n}, x}\left(\hat{\Phi}_{1}^{-1}\left(1+\frac{h}{2} \frac{\left|a_{3}^{n}\right|}{K_{1}\left(U^{n}\right)}\right)^{-1} \delta_{z}\left(K_{1}\left(U^{n}\right) \delta_{\bar{z}} U^{n+1}\right)\right)_{i j k} \\
& -\left[\delta_{a_{1}^{n}, U^{n}, x}\left(\hat{\Phi}_{1}^{-1} \delta_{a_{1}^{n}, U^{n}, y} U^{n+1}\right)_{i j k}+\delta_{a_{1}^{n}, U^{n}, x}\left(\hat{\Phi}_{1}^{-1} \delta_{a_{3}^{n}, U^{n}, z} U^{n+1}\right)_{i j k}\right. \\
& \left.\left.+\delta_{a_{2}^{n}, U^{n}, y}\left(\hat{\Phi}_{1}^{-1} \delta_{a_{3}^{n}, U^{n}, z} U^{n+1}\right)_{i j k}\right]\right\} \\
& -(\Delta t)^{2}\left\{( 1 + \frac { h } { 2 } \frac { | a _ { 1 , i j k } ^ { n } | } { K _ { 1 } ( U ^ { n } ) _ { i j k } } ) ^ { - 1 } \delta _ { x } \left(K _ { 1 } ( U ^ { n } ) \delta _ { \overline { x } } \left(\hat{\Phi}_{1}^{-1}\left(1+\frac{h}{2} \frac{\left|a_{2}^{n}\right|}{K_{1}\left(U^{n}\right)}\right)^{-1}\right.\right.\right. \\
& \times \delta_{y}\left(K_{1}\left(U^{n}\right) \delta_{\bar{y}}\left(\hat{\Phi}_{1}^{-1}\left(1+\frac{h}{2} \frac{\left|a_{3}^{n}\right|}{K_{1}\left(U^{n}\right)}\right)^{-1} \delta_{z}\left(K_{1}\left(U^{n}\right) \delta_{\bar{z}} U^{n+1}\right) \cdots\right)_{i j k}\right. \\
& -\left(1+\frac{h}{2} \frac{\left|a_{1, i j k}^{n}\right|}{K_{1}\left(U^{n}\right) i j k}\right)^{-1} \delta_{x}\left(K _ { 1 } ( U ^ { n } ) \delta _ { \overline { x } } \left(\hat{\Phi}_{1}^{-1}\left(1+\frac{h}{2} \frac{\left|a_{2}^{n}\right|}{K_{1}\left(U^{n}\right)}\right)^{-1}\right.\right. \\
& \times \delta_{y}\left(K_{1}\left(U^{n}\right) \delta_{\bar{y}}\left(\hat{\Phi}_{1}^{-1} \delta_{a_{3}^{n}, U^{n}, z} U^{n}\right) \cdots\right)_{i j k}+\cdots
\end{aligned}
$$




$$
\begin{gathered}
\left.-\delta_{a_{1}^{n}, U^{n}, x}\left(\hat{\Phi}_{1}^{-1} \delta_{a_{2}^{n}, U^{n}, y}\left(\hat{\Phi}_{1}^{-1} \delta_{a_{3}^{n}, U^{n}, z} U^{n+1}\right)\right)_{i j k}\right\} \\
=-K_{2, y, N-1 / 2}^{n} \delta_{\bar{z}} W_{i j, N}^{n+1}+Q\left(U_{i j k}^{n+1}\right), \quad 1 \leqslant i, j, k \leqslant N-1, \\
U_{i j k}^{n+1}=0, \quad\left(x_{i}, y_{j}, z_{k}\right)^{\mathrm{T}} \in \partial \Omega_{1, h} .
\end{gathered}
$$

方程 (10a) (10f) 消去 $V^{n+\frac{1}{3}}$ 和 $V^{n+\frac{2}{3}}$ 可得下述等价的差分方程:

$$
\begin{aligned}
& \hat{\Phi}_{3, i j k} \frac{V_{i j k}^{n+1}-V_{i j k}^{n}}{\Delta t}-\left\{\left(1+\frac{h}{2} \frac{\left|b_{1, i j k}^{n}\right|}{K_{3}\left(V^{n}\right)_{i j k}}\right)^{-1} \delta_{x}\left(K_{3}\left(V^{n}\right) \delta_{\bar{x}}\right)\right. \\
& +\left(1+\frac{h}{2} \frac{\left|b_{2, i j k}^{n}\right|}{K_{3}\left(V^{n}\right)_{i j k}}\right)^{-1} \delta_{y}\left(K_{3}\left(V^{n}\right) \delta_{\bar{y}}\right) \\
& \left.+\left(1+\frac{h}{2} \frac{\left|b_{3, i j k}^{n}\right|}{K_{3}\left(V^{n}\right)_{i j k}}\right)^{-1} \delta_{z}\left(K_{3}\left(V^{n}\right) \delta_{\bar{z}}\right)\right\} V_{i j k}^{n+1} \\
& +\delta_{b_{1}^{n}, V^{n}, x} V_{i j k}^{n+1}+\delta_{b_{2}^{n}, V^{n}, y} V_{i j k}^{n+1}+\delta_{b_{3}^{n}, V^{n}, z} V_{i j k}^{n+1}+\Delta t\left\{\left(1+\frac{h}{2} \frac{\left|b_{1, i j k}^{n}\right|}{K_{3}\left(V^{n}\right)_{i j k}}\right)^{-1}\right. \\
& \times \delta_{x}\left(K_{3}\left(V^{n}\right) \delta_{\bar{x}}\left(\hat{\Phi}_{3}^{-1}\left(1+\frac{h}{2} \frac{\left|b_{2}^{n}\right|}{K_{3}\left(V^{n}\right)}\right)^{-1} \delta_{y}\left(K_{3}\left(V^{n}\right) \delta_{\bar{y}} V^{n+1}\right) \cdot\right)_{i j k}\right. \\
& +\left(1+\frac{h}{2} \frac{\left|b_{1, i j k}^{n}\right|}{K_{3}\left(V^{n}\right)_{i j k}}\right)^{-1} \delta_{x}\left(K _ { 3 } ( V ^ { n } ) \delta _ { \overline { x } } \left(\hat{\Phi}_{3}^{-1}\left(1+\frac{h}{2} \frac{\left|b_{3}^{n}\right|}{K_{3}\left(V^{n}\right)}\right)^{-1}\right.\right. \\
& \left.\times \delta_{z}\left(K_{3}\left(V^{n}\right) \delta_{\bar{z}} V^{n+1}\right) \cdot\right)_{i j k}+\left(1+\frac{h}{2} \frac{\left|b_{2, i j k}^{n}\right|}{K_{3}\left(V^{n}\right)_{i j k}}\right)^{-1} \\
& \times \delta_{y}\left(K_{3}\left(V^{n}\right) \delta_{\bar{y}}\left(\hat{\Phi}_{3}^{-1}\left(1+\frac{h}{2} \frac{\left|b_{3}^{n}\right|}{K_{3}\left(V^{n}\right)}\right)^{-1} \delta_{z}\left(K_{3}\left(V^{n}\right) \delta_{\bar{z}} V^{n+1}\right) \cdot\right)_{i j k}\right\} \\
& -\Delta t\left\{( 1 + \frac { h } { 2 } \frac { | b _ { 1 , i j k } ^ { n } | } { K _ { 3 } ( V ^ { n } ) _ { i j k } } ) ^ { - 1 } \delta _ { x } \left(K_{3}\left(V^{n}\right) \delta_{\bar{x}}\left(\hat{\Phi}_{3}^{-1}\left(\delta_{b_{2}^{n} . V^{n}, y} V^{n+1}\right) \cdot\right)_{i j k}\right.\right. \\
& +\left(1+\frac{h}{2} \frac{\left|b_{1, i j k}^{n}\right|}{K_{3}\left(V^{n}\right)_{i j k}}\right)^{-1} \delta_{x}\left(K_{3}\left(V^{n}\right) \delta_{\bar{x}}\left(\hat{\Phi}_{3}^{-1} \delta_{b_{3}^{n}, V^{n}, z} V^{n+1}\right)\right)_{i j k} \\
& +\delta_{b_{1}^{n}, V^{n}, x}\left(\hat{\Phi}_{3}^{-1}\left(1+\frac{h}{2} \frac{\left|b_{2}^{n}\right|}{K_{3}\left(V^{n}\right)}\right)^{-1} \delta_{y}\left(K_{3}\left(V^{n}\right) \delta_{\bar{y}} V^{n+1}\right)\right)_{i j k} \\
& +\delta_{b_{1}^{n} V^{n}, x}\left(\hat{\Phi}_{3}^{-1}\left(1+\frac{h}{2} \frac{\left|b_{3}^{n}\right|}{K_{3}\left(V^{n}\right)}\right)^{-1} \delta_{z}\left(K_{3}\left(V^{n}\right) \delta_{\bar{z}} V^{n+1}\right)\right)_{i j k} \\
& -\left[\delta_{b^{n}, V^{n}, x}\left(\hat{\Phi}_{3}^{-1} \delta_{b_{2}^{n}, V^{n}, y} V^{n+1}\right)_{i j k}+\delta_{b_{1}^{n}, V^{n}, x}\left(\hat{\Phi}_{3}^{-1} \delta_{a_{3}^{n}, V^{n}, z} V^{n+1}\right)_{i j k}\right. \\
& \left.\left.+\delta_{b_{2}^{n}, V^{n}, y}\left(\hat{\Phi}_{3}^{-1} \delta_{b_{3}^{n}, V^{n}, z} V^{n+1}\right)_{i j k}\right]\right\} \\
& -(\Delta t)^{2}\left\{( 1 + \frac { h } { 2 } \frac { | b _ { 1 , i j k } ^ { n } | } { K _ { 3 } ( V ^ { n } ) _ { i j k } } ) ^ { - 1 } \delta _ { x } \left(K _ { 3 } ( V ^ { n } ) \delta _ { \overline { x } } \left(\hat{\Phi}_{3}^{-1}\left(1+\frac{h}{2} \frac{\left|b_{2}^{n}\right|}{K_{3}\left(V^{n}\right)}\right)^{-1}\right.\right.\right. \\
& \delta_{y}\left(K_{3}\left(V^{n}\right) \delta_{\bar{y}}\left(\hat{\Phi}_{3}^{-1}\left(1+\frac{h}{2} \frac{\left|b_{3}^{n}\right|}{K_{3}\left(V^{n}\right)}\right)^{-1} \delta_{z}\left(K_{3}\left(V^{n}\right) \delta_{\bar{z}} V^{n+1}\right) \cdot\right)_{i j k}\right. \\
& -\left(1+\frac{h}{2} \frac{\left|b_{1, i j k}^{n}\right|}{K_{3}\left(V^{n}\right)_{i j k}}\right)^{-1} \delta_{x}\left(K _ { 3 } ( V ^ { n } ) \delta _ { \overline { x } } \left(\hat{\Phi}_{3}^{-1}\left(1+\frac{h}{2} \frac{\left|b_{2}^{n}\right|}{K_{2}\left(V^{n}\right)}\right)^{-1}\right.\right. \\
& \times \delta_{y}\left(K_{3}\left(V^{n}\right) \delta_{\bar{y}}\left(\hat{\Phi}_{3}^{-1} \delta_{b_{3}^{n}, V^{n}, z} V^{n+1}\right) \cdots\right)_{i j k}+\cdots
\end{aligned}
$$




$$
\begin{gathered}
\left.-\delta_{b_{1}^{n}, V^{n}, x}\left(\hat{\Phi}_{3}^{-1} \delta_{b_{2}^{n}, V^{n}, y}\left(\hat{\Phi}_{3}^{-1} \delta_{b_{3}^{n}, V^{n}, z} V^{n+1}\right)\right)_{i j k}\right\} \\
=K_{2, i j, 1 / 2}^{n} \delta_{z} W_{i j, 0}^{n+1}+Q\left(V_{i j k}^{n+1}\right), \quad 1 \leqslant i, j, k \leqslant N-1,
\end{gathered}
$$

$$
V_{i j k}^{n+1}=0, \quad\left(x_{i}, y_{i}, z_{k}\right)^{\mathrm{T}} \in \partial \Omega_{3, h} .
$$

若问题 (1) (4) 的精确解 $u, v, w$ 是正则的, 则有下述误差方程:

$$
\begin{aligned}
& \hat{\Phi}_{1, i j k} \frac{\xi_{i j k}^{n+1}-\xi_{i j k}^{n}}{\Delta t}-\left\{\left(1+\frac{h}{2} \frac{\left|a_{1, i j k}^{n}\right|}{K_{1}\left(U^{n}\right)_{i j k}}\right)^{-1} \delta_{x}\left(K_{1}\left(U^{n}\right) \delta_{\bar{x}} \xi^{n+1}\right)_{i j k}\right. \\
& +\left(1+\frac{h}{2} \frac{\left|a_{1, i j k}^{n}\right|}{K_{1}\left(U^{n}\right)_{i j k}}\right)^{-1} \delta_{x}\left(\left[K_{1}\left(u^{n+1}\right)-K_{1}\left(U^{n}\right)\right] \delta_{\bar{x}} u^{n+1}\right)_{i j k} \\
& \left.+\left[\left(1+\frac{h}{2} \frac{\left|a_{1, i j k}^{n+1}\right|}{K_{1}\left(u^{n+1}\right)_{i j k}}\right)^{-1}-\left(1+\frac{h}{2} \frac{\left|a_{1, i j k}^{n}\right|}{K_{1}\left(U^{n}\right)_{i j k}}\right)^{-1}\right] \delta_{x}\left(K_{1}\left(u^{n+1}\right) \delta_{\bar{x}} u^{n+1}\right)_{i j k}\right\} \\
& -\left\{\left(1+\frac{h}{2} \frac{\left|a_{2, i j k}^{n}\right|}{K_{1}\left(U^{n}\right)_{i j k}}\right)^{-1} \delta_{y}\left(K_{1}\left(U^{n}\right) \delta_{\bar{y}} \xi^{n+1}\right)_{i j k}\right. \\
& +\left(1+\frac{h}{2} \frac{\left|a_{2, i j k}^{n}\right|}{K_{1}\left(U^{n}\right)_{i j k}}\right)^{-1} \delta_{y}\left(\left[K_{1}\left(u^{n+1}\right)-K_{1}\left(U^{n}\right)\right] \delta_{\bar{y}} u^{n+1}\right)_{i j k} \\
& \left.+\left[\left(1+\frac{h}{2} \frac{\left|a_{2, i j k}^{n+1}\right|}{K_{1}\left(u^{n+1}\right)_{i j k}}\right)^{-1}-\left(1+\frac{h}{2} \frac{\left|a_{2, i j k}^{n+1}\right|}{K_{1}\left(U^{n}\right)_{i j k}}\right)^{-1}\right] \delta_{y}\left(K_{1}\left(u^{n+1}\right) \delta_{\bar{y}} u^{n+1}\right)_{i j k}\right\} \\
& -\left\{\left(1+\frac{h}{2} \frac{\left|a_{3, i j k}^{n}\right|}{K_{1}\left(U^{n}\right)_{i j k}}\right)^{-1} \delta_{z}\left(K_{1}\left(U^{n}\right) \delta_{\bar{z}} \xi^{n+1}\right)_{i j k}\right. \\
& +\left(1+\frac{h}{2} \frac{\left|a_{3, i j k}^{n}\right|}{K_{1}\left(U^{n}\right)_{i j k}}\right)^{-1} \delta_{z}\left(\left[K_{1}\left(u^{n+1}\right)-K_{1}\left(U^{n}\right)\right] \delta_{\bar{z}} u^{n+1}\right)_{i j k} \\
& \left.+\left[\left(1+\frac{h}{2} \frac{\left|a_{3, i j k}^{n+1}\right|}{K_{1}\left(u^{n+1}\right)_{i j k}}\right)^{-1}-\left(1+\frac{h}{2} \frac{\left|a_{3, i j k}^{n}\right|}{K_{1}\left(u^{n}\right)_{i j k}}\right)^{-1}\right] \delta_{z}\left(K_{1}\left(u^{n+1}\right) \delta_{\bar{z}} u^{n+1}\right)_{i j k}\right\} \\
& +\left\{\delta_{a_{1}^{n}, U^{n}, x} \xi_{i j k}^{n+1}+\delta_{a_{1}^{n+1}, u^{n+1}, x} u_{i j k}^{n+1}-\delta_{a_{1}^{n}, U^{n}, x} u_{i j k}^{n+1}\right\} \\
& +\left\{\delta_{a_{2}^{n}, U^{n}, y} \xi_{i j k}^{n+1}+\delta_{a_{2}^{n+1}, u^{n+1}, y} u_{i j k}^{n+1}-\delta_{a_{2}^{n}, U^{n}, y} u_{i j k}^{n+1}\right\} \\
& +\left\{\delta_{a_{3}^{n}, U^{n}, z} \xi_{i j k}^{n+1}+\delta_{a_{3}^{n+1}, u^{n+1}, z} u_{i j k}^{n+1}-\delta_{a_{3}^{n}, U^{n}, z} u_{i j k}^{n+1}\right\} \\
& +\Delta t\left\{( 1 + \frac { h } { 2 } \frac { | a _ { 1 , i j k } ^ { n + 1 } | } { K _ { 1 } ( U ^ { n } ) _ { i j k } } ) ^ { - 1 } \delta _ { x } \left(K _ { 1 } ( U ^ { n } ) \delta _ { \overline { x } } \left(\hat{\Phi}_{1}^{-1}\left(1+\frac{h}{2} \frac{\left|a_{2}^{n}\right|}{K_{1}\left(U^{n}\right)}\right)^{-1}\right.\right.\right. \\
& \left.\times \delta_{y}\left(K_{1}\left(U^{n}\right) \delta_{\bar{y}} \xi^{n+1}\right) \cdot\right)_{i j k}+\left(1+\frac{h}{2} \frac{\left|a_{1, i j k}^{n+1}\right|}{K_{1}\left(U^{n}\right)_{i j k}}\right)^{-1} \delta_{x}\left(K_{1}\left(U^{n}\right)\right. \\
& \times \delta_{\bar{x}}\left(\hat{\Phi}_{1}^{-1}\left(1+\frac{h}{2} \frac{\left|a_{2}^{n}\right|}{K_{1}\left(U^{n}\right)}\right)^{-1} \delta_{y}\left(\left[K_{1}\left(u^{n+1}\right)-K_{1}\left(U^{n}\right)\right] \delta_{\bar{y}} u^{n+1}\right) \cdot\right)_{i j k} \\
& +\left(1+\frac{h}{2} \frac{\left|a_{1, i j k}^{n+1}\right|}{K_{1}\left(U^{n}\right)_{i j k}}\right)^{-1} \delta_{x}\left(K _ { 1 } ( U ^ { n } ) \delta _ { \overline { x } } \left(\hat { \Phi } _ { 1 } ^ { - 1 } \left[\left(1+\frac{h}{2} \frac{\left|a_{2}^{n+1}\right|}{K_{1}\left(u^{n+1}\right)}\right)^{-1}\right.\right.\right. \\
& \left.\left.-\left(1+\frac{h}{2} \frac{\left|a_{2}^{n}\right|}{K_{1}\left(U^{n}\right)}\right)^{-1}\right] \delta_{y}\left(K_{1}\left(u^{n+1}\right) \delta_{\bar{y}} u^{n+1}\right) \cdot\right)_{i j k}
\end{aligned}
$$




$$
\begin{aligned}
& +\left(1+\frac{h}{2} \frac{\left|a_{1, i j k}^{n+1}\right|}{K_{1}\left(U^{n}\right)_{i j k}}\right)^{-1} \delta_{x}\left([ K _ { 1 } ( u ^ { n + 1 } ) - K _ { 1 } ( U ^ { n } ) ] \delta _ { \overline { x } } \left(\hat{\Phi}_{1}^{-1}\left(1+\frac{h}{2} \frac{\left|a_{2}^{n+1}\right|}{K_{1}\left(u^{n+1}\right)}\right)^{-1}\right.\right. \\
& \left.\times \delta_{y}\left(K_{1}\left(u^{n+1}\right) \delta_{\bar{y}} u^{n+1}\right) \cdot\right)_{i j k}+\left[\left(1+\frac{h}{2} \frac{\left|a_{1, i j k}^{n+1}\right|}{K_{1}\left(u^{n+1}\right)_{i j k}}\right)^{-1}-\left(1+\frac{h}{2} \frac{\left|a_{1, i j k}^{n}\right|}{K_{1}\left(U^{n}\right)_{i j k}}\right)^{-1}\right] \\
& \times \delta_{x}\left(K_{1}\left(u^{n+1}\right) \delta_{\bar{x}}\left(\hat{\Phi}_{1}^{-1}\left(1+\frac{h}{2} \frac{\left|a_{2}^{n+1}\right|}{K_{1}\left(U^{n+1}\right)}\right)^{-1} \delta_{y}\left(K_{1}\left(u^{n+1}\right) \delta_{\bar{y}} u^{n+1}\right) \cdot\right)_{i j k}\right. \\
& +\left(1+\frac{h}{2} \frac{\left|a_{1, i j k}^{n}\right|}{K_{1}\left(U^{n}\right)_{i j k}}\right)^{-1} \delta_{x}\left(K _ { 1 } ( U ^ { n } ) \delta _ { \overline { x } } \left(\hat{\Phi}_{1}^{-1}\left(1+\frac{h}{2} \frac{\left|a_{3}^{n}\right|}{K_{1}\left(U^{n}\right)}\right)^{-1}\right.\right. \\
& \left.\times \delta_{z}\left(K_{1}\left(U^{n}\right) \delta_{\bar{z}} \xi_{i j k}^{n+1}\right) \cdot\right)_{i j k}+\left(1+\frac{h}{2} \frac{\left|a_{1, i j k}^{n}\right|}{K_{1}\left(U^{n}\right)_{i j k}}\right)^{-1} \\
& \times \delta_{x}\left(K_{1}\left(U^{n}\right) \delta_{\bar{x}}\left(\hat{\Phi}_{1}^{-1}\left(1+\frac{h}{2} \frac{\left|a_{3}^{n}\right|}{K_{1}\left(U^{n}\right)}\right)^{-1} \delta_{z}\left(\left[K_{1}\left(u^{n+1}\right)-K_{1}\left(U^{n}\right)\right] \delta_{\bar{z}} u^{n+1}\right) \cdot\right)_{i j k}\right. \\
& +\left(1+\frac{h}{2} \frac{\left|a_{1, i j k}^{n}\right|}{K_{1}\left(U^{n}\right)_{i j k}}\right)^{-1} \delta_{x}\left(K _ { 1 } ( U ^ { n } ) \delta _ { \overline { x } } \left(\hat { \Phi } _ { 1 } ^ { - 1 } \left[\left(1+\frac{h}{2} \frac{\left|a_{3}^{n+1}\right|}{K_{1}\left(u^{n+1}\right)}\right)^{-1}\right.\right.\right. \\
& \left.\left.-\left(1+\frac{h}{2} \frac{\left|a_{3}^{n}\right|}{K_{1}\left(U^{n}\right)}\right)^{-1}\right] \delta_{z}\left(K_{1}\left(u^{n+1}\right) \delta_{\bar{z}} u^{n+1}\right) \cdot\right)_{i j k} \\
& +\left[\left(1+\frac{h}{2} \frac{\left|a_{1, i j k}^{n+1}\right|}{K_{1}\left(u^{n+1}\right)_{i j k}}\right)^{-1}-\left(1+\frac{h}{2} \frac{\left|a_{1, i j k}^{n}\right|}{K_{1}\left(U^{n}\right)_{i j k}}\right)^{-1}\right] \\
& \times \delta_{x}\left(K_{1}\left(u^{n+1}\right) \delta_{\bar{x}}\left(\hat{\Phi}_{1}^{-1}\left(1+\frac{h}{2} \frac{\left|a_{2}^{n+1}\right|}{K_{1}\left(u^{n+1}\right)}\right)^{-1} \delta_{z}\left(K_{1}\left(u^{n+1}\right) \delta_{\bar{z}} u^{n+1}\right) \cdot\right)_{i j k}\right. \\
& +\left(1+\frac{h}{2} \frac{\left|a_{2, i j k}^{n}\right|}{K_{1}\left(U^{n}\right)_{i j k}}\right)^{-1} \\
& \times \delta_{y}\left(K_{1}\left(U^{n}\right) \delta_{\bar{y}}\left(\hat{\Phi}_{1}^{-1}\left(1+\frac{h}{2} \frac{\left|a_{3}^{n}\right|}{K_{1}\left(U^{n}\right)}\right)^{-1} \delta_{z}\left(K_{1}\left(U^{n}\right) \delta_{\bar{z}} \xi^{n+1}\right) \cdot\right)_{i j k}+\cdots\right\} \\
& -\Delta t\left\{\left(1+\frac{h}{2} \frac{\left|a_{1, i j k}^{n}\right|}{K_{1}\left(U^{n}\right)_{i j k}}\right)^{-1} \delta_{x}\left(K_{1}\left(U^{n}\right) \delta_{\bar{x}}\left(\hat{\Phi}_{1}^{-1} \delta_{a_{2}^{n}, U^{n}, y} \xi^{n+1}\right)\right)_{i j k}\right. \\
& +\left(1+\frac{h}{2} \frac{\left|a_{1, i j k}^{n}\right|}{K_{1}\left(U^{n}\right)_{i j k}}\right) \delta_{x}\left(K_{1}\left(U^{n}\right) \delta_{\bar{x}}\left(\hat{\Phi}_{1}^{-1}\left[\delta_{a_{2}^{n+1}, u^{n+1}, y}-\delta_{a_{2}^{n}, U^{n}, y}\right] u^{n+1}\right)\right)_{i j k} \\
& +\left(1+\frac{h}{2} \frac{\left|a_{1, i j k}^{n}\right|}{K_{1}\left(U^{n}\right)_{i j k}}\right)^{-1} \delta_{x}\left(\left[K_{1}\left(u^{n+1}\right)-K_{1}\left(U^{n}\right)\right] \delta_{\bar{x}}\left(\hat{\Phi}_{1}^{-1} \delta_{a_{2}^{n+1}, u^{n+1}, y} u^{n+1}\right)\right)_{i j k} \\
& +\left[\left(1+\frac{h}{2} \frac{\left|a_{1, i j k}^{n+1}\right|}{K_{1}\left(u^{n+1}\right)_{i j k}}\right)^{-1}-\left(1+\frac{h}{2} \frac{\left|a_{1, i j k}^{n}\right|}{K_{1}\left(U^{n}\right)_{i j k}}\right)^{-1}\right] \\
& \left.\times \delta_{x}\left(K_{1}\left(u^{n+1}\right) \delta_{\bar{x}}\left(\hat{\Phi}_{1}^{-1} \delta_{a_{2}^{n+1}, u^{n+1}, y} u^{n+1}\right)\right)_{i j k}+\cdots\right\} \\
& -\Delta t\left\{\delta_{a_{1}^{n}, U^{n}, x}\left(\hat{\Phi}_{1}^{-1}\left(1+\frac{h}{2} \frac{\left|a_{2}^{n}\right|}{K_{1}\left(U^{n}\right)}\right)^{-1} \delta_{y}\left(K_{1}\left(U^{n}\right) \delta_{\bar{y}} \xi^{n+1}\right)\right)_{i j k}\right. \\
& +\delta_{a_{1}^{n}, U^{n}, x}\left(\hat{\Phi}_{1}^{-1}\left(1+\frac{h}{2} \frac{\left|a_{2}^{n}\right|}{K_{1}\left(U^{n}\right)}\right)^{-1} \delta_{y}\left(\left[K_{1}\left(u^{n+1}\right)-K_{1}\left(U^{n}\right)\right] \delta_{\bar{y}} u^{n+1}\right)\right)_{i j k} \\
& +\delta_{a_{1}^{n}, U^{n}, x}\left(\hat{\Phi}_{1}^{-1}\left[\left(1+\frac{h}{2} \frac{\left|a_{2}^{n+1}\right|}{K_{1}\left(u^{n+1}\right)}\right)^{-1}-\left(1+\frac{h}{2} \frac{\left|a_{1}^{n}\right|}{K_{1}\left(U^{n}\right)}\right)^{-1}\right]\right.
\end{aligned}
$$




$$
\begin{aligned}
& \left.\times \delta_{y}\left(K_{1}\left(u^{n+1}\right) \delta_{\bar{y}} u^{n+1}\right)\right)_{i j k}+\left[\delta_{a_{1}^{n+1} u^{n+1}, x}-\delta_{a_{1}^{n}, U^{n}, x}\right]\left(\hat{\Phi}_{1}^{-1}\left(1+\frac{h}{2} \frac{\left|a_{1}^{n+1}\right|}{K_{1}\left(u^{n+1}\right)}\right)^{-1}\right. \\
& \left.\left.\times \delta_{y}\left(K_{1}\left(u^{n+1}\right) \delta_{\bar{y}} u^{n+1}\right)\right)_{i j k}+\cdots\right\}+\Delta t\left\{\delta_{a_{1}^{n}, U^{n}, x}\left(\hat{\Phi}_{1}^{-1} \delta_{a_{2}^{n}, U^{n}, y} \xi^{n+1}\right)_{i j k}\right. \\
& +\delta_{a_{1}^{n}, U^{n}, x}\left(\hat{\Phi}_{1}^{-1}\left[\delta_{a_{2}^{n+1}, u^{n+1}, y}-\delta_{a_{2}^{n}, U^{n}, y}\right] u^{n+1}\right)_{i j k} \\
& \left.+\left[\delta_{a_{1}^{n+1}, u^{n+1}, x}-\delta_{a_{1}^{n}, U^{n}, x}\right]\left(\hat{\Phi}_{1}^{-1} \delta_{a_{2}^{n+1}, u^{n+1}, y} u^{n+1}\right)_{i j k}+\cdots\right\} \\
& -(\Delta t)^{2}\left\{( 1 + \frac { h } { 2 } \frac { | a _ { 1 } ^ { n } | } { K _ { 1 } ( U ^ { n } ) } ) ^ { - 1 } \delta _ { x } \left(K _ { 1 } ( U ^ { n } ) \delta _ { \overline { x } } \left(\hat{\Phi}_{1}^{-1}\left(1+\frac{h}{2} \frac{\left|a_{2}^{n}\right|}{K_{1}\left(U^{n}\right)}\right)^{-1}\right.\right.\right. \\
& \times \delta_{y}\left(K_{1}\left(U^{n}\right) \delta_{\bar{y}}\left(\hat{\Phi}_{1}^{-1}\left(1+\frac{h}{2} \frac{\left|a_{3}^{n}\right|}{K_{1}\left(U^{n}\right)}\right)^{-1} \delta_{z}\left(K_{1}\left(U^{n}\right) \delta_{\bar{z}} \xi^{n+1}\right) \cdot\right)_{i j k}+\cdots\right\} \\
& =-K_{2, i j, N-1 / 2}^{n} \delta_{\bar{z}} \omega_{i j, N}^{n+1}+Q_{1}\left(u_{i j k}^{n+1}\right)-Q_{1}\left(U^{n}\right)+\varepsilon_{1, i j k}^{n+1}, \quad 1 \leqslant i, j, k \leqslant N-1,(13 \mathrm{a}) \\
& \xi_{i j k}^{n+1}=0, \quad\left(x_{i}, y_{j}, z_{k}\right)^{\mathrm{T}} \in \partial \Omega_{1},
\end{aligned}
$$

此处

$$
\begin{aligned}
& \left|\varepsilon_{1, i j k}^{n+1}\right| \leqslant M\left\{|| \frac{\partial^{2} u}{\partial t^{2}}\left\|_{L^{\infty}\left(L^{\infty}\right)},\right\| u \|_{L^{\infty}\left(W^{4}, \infty\right)}\right\}\left\{\Delta t+h^{2}\right\} . \\
& \hat{\Phi}_{3, i j k} \frac{\zeta_{i j k}^{n+1}-\zeta_{i j k}^{n}}{\Delta t}-\left\{\left(1+\frac{h}{2} \frac{\left|b_{1, i j k}^{n}\right|}{K_{3}\left(V^{n}\right)_{i j k}}\right)^{-1} \delta_{x}\left(K_{3}\left(V^{n}\right) \delta_{\bar{x}} \zeta^{n+1}\right)_{i j k}\right. \\
& +\left(1+\frac{h}{2} \frac{\left|b_{1, i j k}^{n}\right|}{K_{3}\left(V^{n}\right)_{i j k}}\right)^{-1} \delta_{x}\left(\left[K_{3}\left(v^{n+1}\right)-K_{3}\left(V^{n}\right)\right] \delta_{\bar{x}} v^{n+1}\right)_{i j k} \\
& \left.+\left[\left(1+\frac{h}{2} \frac{\left|b_{1, i j k}^{n+1}\right|}{K_{3}\left(v^{n+1}\right)_{i j k}}\right)^{-1}-\left(1+\frac{h}{2} \frac{\left|b_{1, i j k}^{n}\right|}{K_{3}\left(V^{n}\right)_{i j k}}\right)^{-1}\right] \delta_{x}\left(K_{3}\left(v^{n+1}\right) \delta_{\bar{x}} v^{n+1}\right)_{i j k}\right\} \\
& +\left\{\left(1+\frac{h}{2} \frac{\left|b_{2, i j k}^{n}\right|}{K_{3}\left(V^{n}\right)_{i j k}}\right)^{-1} \delta_{y}\left(K_{3}\left(V^{n}\right) \delta_{\bar{y}} \xi^{n+1}\right)_{i j k}\right. \\
& +\left(\left(1+\frac{h}{2} \frac{\left|b_{2, i j k}^{n}\right|}{K_{3}\left(V^{n}\right)_{i j k}}\right)^{-1} \delta_{y}\left(\left[K_{3}\left(v^{n+1}\right)-K_{3}\left(V^{n}\right)\right] \delta_{\bar{y}} v^{n+1}\right)_{i j k}\right. \\
& \left.+\left[\left(1+\frac{h}{2} \frac{\left|b_{2, i j k}^{n+1}\right|}{K_{3}\left(v^{n+1}\right)_{i j k}}\right)^{-1}-\left(1+\frac{h}{2} \frac{\left|b_{2, i j k}^{n}\right|}{K_{3}\left(V^{n}\right)_{i j k}}\right)^{-1}\right] \delta_{y}\left(K_{3}\left(v^{n+1}\right) \delta_{\bar{y}} v^{n+1}\right)_{i j k}\right\} \\
& +\left\{\left(1+\frac{h}{2} \frac{\left|b_{3, i j k}^{n}\right|}{K_{3}\left(V^{n}\right)_{i j k}}\right)^{-1} \delta_{z}\left(K_{3}\left(V^{n}\right) \delta_{\bar{z}} \zeta^{n+1}\right)_{i j k}\right. \\
& +\left\{\delta_{b_{3}^{n}, V^{n}, z} \zeta_{i j k}^{n+1}+\delta_{b_{3}^{n+1}, v^{n+1}, z} v_{i j k}^{n+1}-\delta_{b_{3}^{n}, V^{n}, z} v_{i j k}^{n+1}\right\} \\
& +\left(1+\frac{h}{2} \frac{\left|b_{3, i j k}^{n}\right|}{K_{3}\left(V^{n}\right)_{i j k}}\right)^{-1} \delta_{z}\left(\left[K_{3}\left(v^{n+1}\right)-K_{3}\left(V^{n}\right)\right] \delta_{\bar{z}} v^{n+1}\right)_{i j k} \\
& \left.+\left[\left(1+\frac{h}{2} \frac{\left|b_{3, i j k}^{n+1}\right|}{K_{3}\left(v^{n+1}\right)_{i j k}}\right)^{-1}-\left(1+\frac{h}{2} \frac{\left|b_{3, i j k}^{n}\right|}{K_{3}\left(V^{n}\right)_{i j k}}\right)^{-1}\right] \delta_{z}\left(K_{3}\left(v^{n+1}\right) \delta_{\bar{z}} v^{n+1}\right)_{i j k}\right\} \\
& +\left\{\delta_{b_{2}^{n}, V^{n}, y} \zeta_{i j k}^{n+1}+\delta_{b_{2}^{n+1}, v^{n+1}, y} v_{i j k}^{n+1}-\delta_{b_{2}^{n}, V^{n}, y} v_{i j k}^{n+1}\right\} \\
& +
\end{aligned}
$$




$$
\begin{aligned}
& +\Delta t\left\{( 1 + \frac { h } { 2 } \frac { | b _ { 1 , i j k } ^ { n } | } { K _ { 3 } ( V ^ { n } ) _ { i j k } } ) ^ { - 1 } \delta _ { x } \left(K _ { 3 } ( V ^ { n } ) \delta _ { \overline { x } } \left(\hat{\Phi}_{3}^{-1}\left(1+\frac{h}{2} \frac{\left|b_{2}^{n}\right|}{K_{3}\left(V^{n}\right)}\right)^{-1}\right.\right.\right. \\
& \left.\times \delta_{y}\left(K_{3}\left(V^{n}\right) \delta_{\bar{y}} \zeta^{n+1}\right) \cdot\right)_{i j k}+\left(1+\frac{h}{2} \frac{\left|b_{1, i j k}^{n}\right|}{K_{3}\left(V^{n}\right)_{i j k}}\right)^{-1} \\
& \times \delta_{x}\left(K_{3}\left(V^{n}\right) \delta_{\bar{x}}\left(\hat{\Phi}_{3}^{-1}\left(1+\frac{h}{2} \frac{\left|b_{2}^{n}\right|}{K_{3}\left(V^{n}\right)}\right)^{-1} \delta_{y}\left(\left[K_{3}\left(v^{n+1}\right)-K_{3}\left(V^{n}\right)\right] \delta_{\bar{y}} v^{n+1}\right) \cdot\right)_{i j k}\right. \\
& +\left(1+\frac{h}{2} \frac{\left|b_{1, i j k}^{n}\right|}{K_{3}\left(V^{n}\right)_{i j k}}\right)^{-1} \delta_{x}\left(K _ { 3 } ( V ^ { n } ) \delta _ { \overline { x } } \left(\hat { \Phi } _ { 3 } ^ { - 1 } \left[\left(1+\frac{h}{2} \frac{\left|b_{2}^{n+1}\right|}{K_{3}\left(v^{n+1}\right)}\right)^{-1}\right.\right.\right. \\
& \left.\left.-\left(1+\frac{h}{2} \frac{\left|b_{2}^{n}\right|}{K_{3}\left(V^{n}\right)}\right)^{-1}\right] \delta_{y}\left(K_{3}\left(v^{n+1}\right) \delta_{\bar{y}} v^{n+1}\right) \cdot\right)_{i j k} \\
& +\left(1+\frac{h}{2} \frac{\left|b_{1, i j k}^{n}\right|}{K_{3}\left(V^{n}\right)_{i j k}}\right)^{-1} \delta_{x}\left([ K _ { 3 } ( v ^ { n + 1 } ) - K _ { 3 } ( V ^ { n } ) ] \delta _ { \overline { x } } \left(\hat{\Phi}_{3}^{-1}\left(1+\frac{h}{2} \frac{\left|b_{2}^{n+1}\right|}{K_{3}\left(v^{n+1}\right)}\right)^{-1}\right.\right. \\
& \left.\times \delta_{y}\left(K_{3}\left(v^{n+1}\right) \delta_{\bar{y}} v^{n+1}\right) \cdot\right)_{i j k}+\left[\left(1+\frac{h}{2} \frac{\left|b_{1, i j k}^{n}\right|}{K_{3}\left(v^{n+1}\right)_{i j k}}\right)^{-1}-\left(1+\frac{h}{2} \frac{\left|b_{1, i j k}^{n}\right|}{K_{3}\left(V^{n}\right)_{i j k}}\right)^{-1}\right] \\
& \times \delta_{\bar{x}}\left(K_{3}\left(v^{n+1}\right) \delta_{\bar{x}}\left(\hat{\Phi}_{3}^{-1}\left(1+\frac{h}{2} \frac{\left|b_{2}^{n+1}\right|}{K_{3}\left(v^{n+1}\right)}\right)^{-1} \delta_{y}\left(K_{3}\left(v^{n+1}\right) \delta_{\bar{y}} v^{n+1}\right) \cdot\right)_{i j k}+\cdots\right\} \\
& -\Delta t\left\{\left(1+\frac{h}{2} \frac{\left|b_{1, i j k}^{n}\right|}{K_{3}\left(V^{n}\right)_{i j k}}\right)^{-1} \delta_{x}\left(K_{3}\left(V^{n}\right) \delta_{\bar{x}}\left(\hat{\Phi}_{3}^{-1} \delta_{b_{2}^{n}, V^{n}, y} \zeta^{n+1}\right)\right)_{i j k}\right. \\
& +\left(1+\frac{h}{2} \frac{\left|b_{1, i j k}^{n}\right|}{K_{3}\left(V^{n}\right)_{i j k}}\right)^{-1} \delta_{x}\left(K_{3}\left(V^{n}\right) \delta_{\bar{x}}\left(\hat{\Phi}_{3}^{-1} \delta_{b_{2}^{n+1}, v^{n+1}, y} v^{n+1}-\delta_{b_{2}^{n}, V^{n}, y} v^{n+1}\right)\right)_{i j k} \\
& +\left(1+\frac{h}{2} \frac{\left|b_{1, i j k}^{n}\right|}{K_{3}\left(V^{n}\right)_{i j k}}\right)^{-1} \delta_{x}\left(\left[K_{3}\left(v^{n+1}\right)-K_{3}\left(V^{n}\right)\right] \delta_{\bar{x}}\left(\hat{\Phi}_{3}^{-1} \delta_{b_{2}^{n+1}, v^{n+1}, y} v^{n+1}\right)\right)_{i j k} \\
& +\left[\left(1+\frac{h}{2} \frac{\left|b_{1, i j k}^{n+1}\right|}{K_{3}\left(v^{n+1}\right)_{i j k}}\right)^{-1}-\left(1+\frac{h}{2} \frac{\left|b_{1, i j k}^{n}\right|}{K_{3}\left(V^{n}\right)_{i j k}}\right)^{-1}\right] \\
& \left.\times \delta_{x}\left(K_{3}\left(v^{n+1}\right) \delta_{\bar{x}}\left(\hat{\Phi}_{3}^{-1} \delta_{b_{2}^{n+1}, v^{n+1}, y} v^{n+1}\right)\right)_{i j k}+\cdots\right\} \\
& -\Delta t\left\{\delta_{b_{1}^{n}, V^{n}, x}\left(\hat{\Phi}_{3}^{-1}\left(1+\frac{h}{2} \frac{\left|b_{2}^{n}\right|}{K_{3}\left(V^{n}\right)}\right)^{-1} \delta_{y}\left(K_{3}\left(V^{n}\right) \delta_{\bar{y}} \zeta^{n+1}\right)\right)_{i j k}\right. \\
& +\delta_{b_{1}^{n}, V^{n}, x}\left(\hat{\Phi}_{3}^{-1}\left(1+\frac{h}{2} \frac{\left|b_{2}^{n}\right|}{K_{3}\left(V^{n}\right)}\right)^{-1} \delta_{y}\left(\left[K_{3}\left(v^{n+1}\right)-K_{3}\left(V^{n}\right)\right] \delta_{\bar{y}} v^{n+1}\right)\right)_{i j k} \\
& +\delta_{b_{1}^{n}, V^{n}, x}\left(\hat{\Phi}_{3}^{-1}\left[\left(1+\frac{h}{2} \frac{\left|b_{2}^{n+1}\right|}{K_{3}\left(v^{n+1}\right)}\right)^{-1}-\left(1+\frac{h}{2} \frac{\left|b_{2}^{n}\right|}{K_{3}\left(V^{n}\right)}\right)^{-1}\right]\right. \\
& \left.\times \delta_{y}\left(K\left(v^{n+1}\right) \delta_{\bar{y}} v^{n+1}\right)\right)_{i j k}+\left[\delta_{b_{1}^{n+1}, v^{n+1}, x}-\delta_{b_{1}^{n}, V^{n}, x}\right]\left(\hat{\Phi}_{3}^{-1}\left(1+\frac{h}{2} \frac{\left|a_{2}^{n+1}\right|}{K_{3}\left(v^{n+1}\right)}\right)^{-1}\right. \\
& \left.\left.\times \delta_{y}\left(K_{3}\left(v^{n+1}\right) \delta_{\bar{y}} v^{n+1}\right)\right)_{i j k}+\cdots\right\}+\Delta t\left\{\delta_{b_{1}^{n}, V^{n}, x}\left(\hat{\Phi}_{3}^{-1} \delta_{b_{2}^{n}, V^{n}, y} \zeta^{n+1}\right)_{i j k}\right. \\
& +\delta_{b_{1}^{n} V^{n}, x}\left(\hat{\Phi}_{1}^{-1}\left[\delta_{b_{2}^{n+1}, v^{n+1}, y}-\delta_{b_{2}^{n}, V^{n}, y}\right] v^{n+1}\right)_{i j k} \\
& \left.+\left[\delta_{b_{1}^{n+1}, v^{n+1}, x}-\delta_{b_{1}^{n}, V^{n}, x}\right]\left(\hat{\Phi}_{3}^{-1} \delta_{b_{2}^{n+1}, v^{n+1}, y} v^{n+1}\right)_{i j k}+\cdots\right\} \\
& =K_{2, i j, 1 / 2}^{n} \delta_{z} \omega_{i j, 0}^{n+1}+Q_{3}\left(v_{i j k}^{n+1}\right)-Q_{3}\left(V_{i j k}^{n+1}\right)+\varepsilon_{3, i j k}^{n+1}, \quad 1 \leqslant i, j, k \leqslant N-1 \text {, }
\end{aligned}
$$




$$
\xi_{i j k}^{n+1}=0, \quad\left(x_{i}, y_{j}, z_{k}\right)^{\mathrm{T}} \in \partial \Omega_{3, h},
$$

此处

$$
\begin{gathered}
\left|\varepsilon_{3, i j k}^{n+1}\right| \leqslant M\left\{\left\|\frac{\partial^{2} v}{\partial t^{2}}\right\|_{L^{\infty}\left(L^{\infty}\right)},\|v\|_{L^{\infty}\left(W^{4}, \infty\right)}\right\}\left\{\Delta t+h^{2}\right\} . \\
\Phi_{2, i j k} \frac{\omega_{i j k}^{n+1}-\omega_{i j k}^{n}}{\Delta t}=\delta_{z}\left(K_{2}^{n} \delta_{\bar{z}} \omega^{n+1}\right)_{i j k}+\varepsilon_{2, i j k}^{n+1}, \quad 1 \leqslant i, j, k \leqslant n-1,
\end{gathered}
$$

此处

$$
\left|\varepsilon_{2, i j k}^{n+1}\right| \leqslant M\left\{\left\|\frac{\partial^{2} w}{\partial t^{2}}\right\|_{L^{\infty}\left(L^{\infty}\right)},\|w\|_{L^{\infty}\left(W^{4}, \infty\right)}\right\}\left\{\Delta t+h^{2}\right\} .
$$

对方程 (13a)、(14a) 和 (15) 分别乘以 $2 \Delta t \xi_{i j k}^{n+1}, 2 \Delta t \zeta_{i j k}^{n+1}$ 和 $2 \Delta t \omega_{i j k}^{n+1}$ 后分别 在 $\Omega_{1, h}, \Omega_{3, h}$, 和 $\Omega_{2, h}$ 上作内积, 分部求和并利用 (13b) 、(14b) 和 (3b) 式可得

$$
\begin{aligned}
& \left\{\left\|\hat{\Phi}_{1}^{1 / 2} \xi^{n+1}\right\|^{2}-\left\|\hat{\Phi}_{1}^{1 / 2} \xi^{n}\right\|^{2}\right\}+(\Delta t)^{2}\left\|\hat{\Phi}_{1}^{1 / 2} d_{t} \xi^{n}\right\|^{2} \\
& +2 \Delta t\left\{\left\langle K_{1}\left(U^{n}\right) \delta_{\bar{x}} \xi^{n+1}, \delta_{\bar{x}}\left(\left(1+\frac{h}{2} \frac{\left|a_{1}^{n}\right|}{K_{1}\left(U^{n}\right)}\right)^{-1} \xi^{n+1}\right)\right\rangle\right. \\
& +\left\langle\left[K_{1}\left(u^{n+1}\right)-K_{1}\left(U^{n}\right)\right] \delta_{\bar{x}} u^{n+1}, \delta_{\bar{x}}\left(\left(1+\frac{h}{2} \frac{\left|a_{1}^{n}\right|}{K_{1}\left(U^{n}\right)}\right)^{-1} \xi^{n+1}\right)\right\rangle \\
& \left.+\left\langle\left[\left(1+\frac{h}{2} \frac{\left|a_{1}^{n+1}\right|}{K_{1}\left(u^{n+1}\right)}\right)^{-1}-\left(1+\frac{h}{2} \frac{\left|a_{1}^{n}\right|}{K_{1}\left(U^{n}\right)}\right)^{-1}\right] \delta_{x}\left(K_{1}\left(u^{n+1}\right) \delta_{\bar{x}} u^{n+1}\right), \xi^{n+1}\right\rangle\right\} \\
& +2 \Delta t\left\{\left\langle K_{1}\left(U^{n}\right) \delta_{\bar{y}} \xi^{n+1}, \delta_{\bar{y}}\left(\left(1+\frac{h}{2} \frac{\left|a_{2}^{n}\right|}{K_{1}\left(U^{n}\right)}\right)^{-1} \xi^{n+1}\right)\right\rangle\right. \\
& +\left\langle\left[K_{1}\left(u^{n+1}\right)-K_{1}\left(U^{n}\right)\right] \delta_{\bar{y}} u^{n+1}, \delta_{\bar{y}}\left(\left(1+\frac{h}{2} \frac{\left|a_{2}^{n}\right|}{K_{1}\left(U^{n}\right)}\right)^{-1} \xi^{n+1}\right)\right\rangle \\
& \left.+\left\langle\left[\left(1+\frac{h}{2} \frac{\left|a_{2}^{n+1}\right|}{K_{1}\left(u^{n+1}\right)}\right)^{-1}-\left(1+\frac{h}{2} \frac{\left|a_{1}^{n}\right|}{K_{1}\left(U^{n}\right)}\right)^{-1}\right] \delta_{y}\left(K_{1}\left(u^{n+1}\right) \delta_{\bar{y}} u^{n+1}\right), \xi^{n+1}\right\rangle\right\} \\
& +2 \Delta t\left\{\left\langle K_{1}\left(U^{n}\right) \delta_{\bar{z}} \xi^{n+1}, \delta_{\bar{z}}\left(\left(1+\frac{h}{2} \frac{\left|a_{3}^{n}\right|}{K_{1}\left(U^{n}\right)}\right)^{-1} \xi^{n+1}\right)\right\rangle\right. \\
& +\left\langle\left[K_{1}\left(u^{n+1}\right)-K_{1}\left(U^{n}\right)\right] \delta_{\bar{z}} u^{n+1}, \delta_{\bar{z}}\left(\left(1+\frac{h}{2} \frac{\left|a_{3}^{n}\right|}{K_{1}\left(U^{n}\right)}\right)^{-1} \xi^{n+1}\right)\right\rangle \\
& \left.+\left\langle\left[\left(1+\frac{h}{2} \frac{\left|a_{3}^{n+1}\right|}{K_{1}\left(u^{n+1}\right)}\right)^{-1}-\left(1+\frac{h}{2} \frac{\left|a_{3}^{n}\right|}{K_{1}\left(U^{n}\right)}\right)^{-1}\right] \delta_{z}\left(K_{1}\left(u^{n+1}\right) \delta_{\bar{z}} u^{n+1}\right), \xi^{n+1}\right\rangle\right\} \\
& =-2 \Delta t\left\{\left\langle\delta_{a_{1}^{n}, U^{n}, x} \xi^{n+1}, \xi^{n+1}\right\rangle+\left\langle\left[\delta_{a_{1}^{n+1}, u^{n+1}, x}-\delta_{a_{1}^{n}, U^{n}, x}\right] u^{n+1}, \xi^{n+1}\right\rangle\right\} \\
& -2 \Delta t\left\{\left\langle\delta_{a_{2}^{n}, U^{n}, y} \xi^{n+1}, \xi^{n+1}\right\rangle+\left\langle\left[\delta_{a_{2}^{n+1}, u^{n+1}, y}-\delta_{a_{2}^{n}, U^{n}, y}\right] u^{n+1}, \xi^{n+1}\right\rangle\right\} \\
& -2 \Delta t\left\{\left\langle\delta_{a_{3}^{n}, U^{n}, z} \xi^{n+1}, \xi^{n+1}\right\rangle+\left\langle\left[\delta_{a_{3}^{n+1}, u^{n+1}, z}-\delta_{a_{3}^{n}, U^{n}, z}\right] u^{n+1}, \xi^{n+1}\right\rangle\right\} \\
& -2(\Delta t)^{2}\left\{\left\langle\left(1+\frac{h}{2} \frac{\left|a_{1}^{n}\right|}{K_{1}\left(U^{n}\right)}\right)^{-1}\right.\right. \\
& \times \delta_{x}\left(K_{1}\left(U^{n}\right) \delta_{\bar{x}}\left(\hat{\Phi}_{1}^{-1}\left(1+\frac{h}{2} \frac{\left|a_{2}^{n}\right|}{K_{1}\left(U^{n}\right)}\right)^{-1} \delta_{y}\left(K_{1}\left(U^{n}\right) \delta_{\bar{y}} \xi^{n+1}\right) \cdot\right), \xi^{n+1}\right\rangle
\end{aligned}
$$




$$
\begin{aligned}
& +\left\langle( 1 + \frac { h } { 2 } \frac { | a _ { 1 } ^ { n } | } { K _ { 1 } ( U ^ { n } ) } ) ^ { - 1 } \delta _ { x } \left(K _ { 1 } ( U ^ { n } ) \delta _ { \overline { x } } \left(\hat{\Phi}_{1}^{-1}\left(1+\frac{h}{2} \frac{\left|a_{2}^{n}\right|}{K_{1}\left(U^{n}\right)}\right)^{-1}\right.\right.\right. \\
& \left.\left.\times \delta_{y}\left(\left[K_{1}\left(u^{n+1}\right)-K_{1}\left(U^{n}\right)\right] \delta_{\bar{y}} u^{n+1}\right) \cdot\right), \xi^{n+1}\right\rangle+\cdots \\
& +\left\langle\left[\left(1+\frac{h}{2} \frac{\left|a_{1}^{n+1}\right|}{K_{1}\left(u^{n+1}\right)}\right)^{-1}-\left(1+\frac{h}{2} \frac{\left|a_{1}^{n}\right|}{K_{1}\left(U^{n}\right)}\right)^{-1}\right]\right. \\
& \left.\times \delta_{x}\left(K_{1}\left(u^{n+1}\right) \delta_{\bar{x}}\left(\hat{\Phi}_{1}^{-1}\left(1+\frac{h}{2} \frac{\left|a_{2}^{n+1}\right|}{K_{1}\left(u^{n+1}\right)}\right)^{-1} \delta_{y}\left(K_{1}\left(u^{n+1}\right) \delta_{\bar{y}} u^{n+1}\right) \cdot\right), \xi^{n+1}\right\rangle\right\} \\
& -2(\Delta t)^{2}\left\{\left\langle\left(1+\frac{h}{2} \frac{\left|a_{1}^{n}\right|}{K_{1}\left(U^{n}\right)}\right)^{-1}\right.\right. \\
& \left.\times \delta_{x}\left(K_{1}\left(U^{n}\right) \delta_{\bar{x}}\left(\hat{\Phi}_{1}^{-1}\left(1+\frac{h}{2} \frac{\left|a_{3}^{n}\right|}{K_{1}\left(U^{n}\right)}\right)^{-1} \delta_{z}\left(K_{1}\left(U^{n}\right) \delta_{\bar{z}} \xi^{n+1}\right) \cdot\right), \xi\right\rangle+\cdots\right\} \\
& -2(\Delta t)^{2}\left\{\left\langle\left(1+\frac{h}{2} \frac{\left|a_{2}^{n}\right|}{K_{1}\left(U^{n}\right)}\right)^{-1}\right.\right. \\
& \left.\times \delta_{y}\left(K_{1}\left(U^{n}\right) \delta_{\bar{y}}\left(\hat{\Phi}_{1}^{-1}\left(1+\frac{h}{2} \frac{\left|a_{3}^{n}\right|}{K_{1}\left(U^{n}\right)}\right)^{-1} \delta_{z}\left(K_{1}\left(U^{n}\right) \delta_{\bar{z}} \xi^{n+1}\right) \cdot\right), \xi^{n+1}\right\rangle+\cdots\right\} \\
& +2(\Delta t)^{2}\left\{\left\langle\left(1+\frac{h}{2} \frac{\left|a_{1}^{n}\right|}{K_{1}\left(U^{n}\right)}\right)^{-1} \delta_{x}\left(K_{1}\left(U^{n}\right) \delta_{\bar{x}}\left(\hat{\Phi}_{1}^{-1} \delta_{a_{2}^{n}, U^{n}, y} \xi^{n+1}\right) \cdot\right), \xi^{n+1}\right\rangle\right. \\
& +\left\langle\left(1+\frac{h}{2} \frac{\left|a_{1}^{n}\right|}{K_{1}\left(U^{n}\right)}\right)^{-1} \delta_{x}\left(K_{1}\left(U^{n}\right) \delta_{\bar{x}}\left(\hat{\Phi}_{1}^{-1}\left[\delta_{a_{2}^{n+1}, u^{n+1}, y}-\delta_{a^{n} U^{n}, y}\right] u^{n+1}\right)\right), \xi^{n+1}\right\rangle \\
& +\cdots+\left\langle\left[\left(1+\frac{h}{2} \frac{\left|a_{1}^{n+1}\right|}{K_{1}\left(u^{n+1}\right)}\right)^{-1}-\left(1+\frac{h}{2} \frac{\left|a_{1}^{n}\right|}{K_{1}\left(U^{n}\right)}\right)^{-1}\right]\right. \\
& \left.\left.\times \delta_{x}\left(K_{1}\left(u^{n+1}\right) \delta_{\bar{x}}\left(\hat{\Phi}_{1}^{-1} \delta_{a_{2}^{n+1}, u^{n+1}, y} u^{n+1}\right)\right), \xi^{n+1}\right\rangle+\cdots\right\} \\
& +2(\Delta t)^{2}\left\{\left\langle\delta_{a_{1}^{n}, U^{n}, x}\left(\hat{\Phi}_{1}^{-1}\left(1+\frac{h}{2} \frac{\left|a_{2}^{n}\right|}{K_{1}\left(U^{n}\right)}\right)^{-1} \delta_{y}\left(K_{1}\left(U^{n}\right) \delta_{\bar{y}} \xi^{n+1}\right)\right), \xi^{n+1}\right\rangle\right. \\
& +\left\langle\delta_{a_{1}^{n}, U^{n}, x}\left(\hat{\Phi}_{1}^{-1}\left(1+\frac{h}{2} \frac{\left|a_{2}^{n}\right|}{K_{1}\left(U^{n}\right)}\right)^{-1} \delta_{y}\left(\left[K_{1}\left(u^{n+1}\right)-K_{1}\left(U^{n}\right)\right] \delta_{\bar{y}} u^{n+1}\right)\right), \xi^{n+1}\right\rangle \\
& +\cdots+\left\langle[ \delta _ { a _ { 1 } ^ { n + 1 } , u ^ { n + 1 } , x } - \delta _ { a _ { 1 } ^ { n } , U ^ { n } , x } ] \left(\hat{\Phi}_{1}^{-1}\left(1+\frac{h}{2} \frac{\left|a_{2}^{n+1}\right|}{K_{1}\left(u^{n+1}\right)}\right)^{-1}\right.\right. \\
& \left.\left.\left.\times \delta_{y}\left(K_{1}\left(u^{n+1}\right) \delta_{\bar{y}} u^{n+1}\right)\right), \xi^{n+1}\right\rangle+\cdots\right\} \\
& -2(\Delta t)^{2}\left\{\left\langle\delta_{a_{1}^{n}, U^{n}, x}\left(\hat{\Phi}_{1}^{-1} \delta_{a_{2}^{n}, U^{n}, y} \xi^{n+1}\right), \xi^{n+1}\right\rangle\right. \\
& +\left\langle\delta_{a_{1}^{n}, U^{n}, x}\left(\hat{\Phi}_{1}^{-1}\left[\delta_{a_{2}^{n+1}, u^{n+1}, y}-\delta_{a_{2}^{n}, U^{n}, y}\right] u^{n+1}\right), \xi^{n+1}\right\rangle \\
& \left.+\left\langle\left[\delta_{a_{1}^{n+1}, u^{n+1}, x}-\delta_{a_{1}^{n}, U^{n}, x}\right]\left(\hat{\Phi}_{1}^{-1} \delta_{a_{2}^{n+1}, u^{n+1}, y} u^{n+1}\right), \xi^{n+1}\right\rangle\right\} \\
& +2(\Delta t)^{3}\left\{\left\langle( 1 + \frac { h } { 2 } \frac { | a _ { 1 } ^ { n } | } { K _ { 1 } ( U ^ { n } ) } ) ^ { - 1 } \delta _ { x } \left(K _ { 1 } ( U ^ { n } ) \delta _ { \overline { x } } \left(\hat{\Phi}_{1}^{-1}\left(1+\frac{h}{2} \frac{\left|a_{2}^{n}\right|}{K_{1}\left(U^{n}\right)}\right)^{-1}\right.\right.\right.\right. \\
& \left.\times \delta_{y}\left(K_{1}\left(U^{n}\right) \delta_{\bar{y}}\left(\hat{\Phi}_{1}^{-1}\left(1+\frac{h}{2} \frac{\left|a_{3}^{n}\right|}{K_{1}\left(U^{n}\right)}\right)^{-1} \delta_{z}\left(K_{1}\left(U^{n}\right) \delta_{\bar{z}} \xi^{n+1}\right) \cdot\right), \xi^{n+1}\right\rangle+\cdots\right\} \\
& =-2 \Delta t \sum_{i, j=1}^{N-1} K_{2, i j, N-\frac{1}{2}}^{n} \delta_{\bar{z}} \omega_{i j, N}^{n+1} \xi_{i j, 0}^{n+1} h^{2}
\end{aligned}
$$




$$
\begin{aligned}
& +2 \Delta t\left\langle Q_{1}\left(u^{n+1}\right)-Q_{1}\left(U^{n}\right), \xi^{n+1}\right\rangle+\Delta t\left\langle\varepsilon_{1}^{n+1}, \xi^{n+1}\right\rangle . \\
& \left\{\left\|\Phi_{3}^{1 / 2} \zeta^{n+1}\right\|^{2}-\left\|\hat{\Phi}_{3}^{1 / 2} \zeta^{n}\right\|^{2}\right\}+(\Delta t)^{2}\left\|\hat{\Phi}_{3}^{1 / 2} d_{1} \zeta^{n}\right\|^{2} \\
& +2 \Delta t\left\{\left\langle K_{3}\left(V^{n}\right) \delta_{\bar{x}} \zeta^{n+1}, \delta_{\bar{x}}\left(\left(1+\frac{h}{2} \frac{\left|b_{1}^{n}\right|}{K_{3}\left(U^{n}\right)}\right)^{-1} \zeta^{n+1}\right)\right\rangle\right. \\
& +\left\langle\left[K_{3}\left(v^{n+1}\right)-K_{3}\left(V^{n}\right)\right] \delta_{\bar{x}} v^{n+1}, \delta_{\bar{x}}\left(\left(1+\frac{h}{2} \frac{\left|b_{1}^{n}\right|}{K_{3}\left(V^{n}\right)}\right)^{-1} \zeta^{n+1}\right)\right\rangle \\
& \left.+\left\langle\left[\left(1+\frac{h}{2} \frac{\left|b_{1}^{n+1}\right|}{K_{3}\left(v^{n+1}\right)}\right)^{-1}-\left(1+\frac{h}{2} \frac{\left|b_{1}^{n}\right|}{K_{3}\left(V^{n}\right)}\right)^{-1}\right] \delta_{x}\left(K_{3}\left(v^{n+1}\right) \delta_{\bar{x}} v^{n+1}\right), \zeta^{n+1}\right\rangle\right\} \\
& +2 \Delta t\left\{\left\langle K_{3}\left(V^{n}\right) \delta_{\bar{y}} \zeta^{n+1}, \delta_{\bar{y}}\left(\left(1+\frac{h}{2} \frac{\left|b_{2}^{n}\right|}{K_{3}\left(V^{n}\right)}\right)^{-1} \zeta^{n+1}\right)\right\rangle\right. \\
& +\left\langle\left[K_{3}\left(v^{n+1}\right)-K_{3}\left(V^{n}\right)\right] \delta_{\bar{y}} v^{n+1}, \delta_{\bar{y}}\left(\left(1+\frac{h}{2} \frac{\left|b_{1}^{n}\right|}{K_{3}\left(V^{n}\right)}\right)^{-1} \zeta^{n+1}\right)\right\rangle \\
& \left.+\left\langle\left[\left(1+\frac{h}{2} \frac{\left|b_{2}^{n}\right|}{K_{3}\left(v^{n+1}\right)}\right)^{-1}-\left(1+\frac{h}{2} \frac{\left|b_{1}^{n}\right|}{K_{3}\left(V^{n}\right)}\right)^{-1}\right] \delta_{y}\left(K_{3}\left(v^{n+1}\right) \delta_{\bar{y}} v^{n+1}\right), \zeta^{n+1}\right\rangle\right\} \\
& +2 \Delta t\left\{\left\langle K_{3}\left(V^{n}\right) \delta_{\bar{z}} \zeta^{n+1}, \delta_{\bar{z}}\left(\left(1+\frac{h}{2} \frac{\left|b_{3}^{n}\right|}{K_{3}\left(V^{n}\right)}\right)^{-1} \zeta^{n+1}\right)\right\rangle\right. \\
& +\left\langle\left[K_{3}\left(v^{n+1}\right)-K_{3}\left(V^{n}\right)\right] \delta_{\bar{z}} v^{n+1}, \delta_{\bar{z}}\left(\left(1+\frac{h}{2} \frac{\left|b_{3}^{n}\right|}{K_{3}\left(V^{n}\right)}\right)^{-1} \zeta^{n+1}\right)\right\rangle \\
& +\left\langle\left[\left(1+\frac{h}{2} \frac{\left|b_{3}^{n}\right|}{K_{3}\left(v^{n+1}\right)}\right)^{-1}-\left(1+\frac{h}{2} \frac{\left|b_{3}^{n}\right|}{K_{3}\left(V^{n}\right)}\right)^{-1}\right]\right. \\
& \left.\left.\times \delta_{z}\left(K_{3}\left(v^{n+1}\right) \delta_{\bar{z}} v^{n+1}\right), \zeta^{n+1}\right\rangle\right\} \\
& =-2 \Delta t\left\{\left\langle\delta_{b_{1}^{n}, V^{n}, x} \zeta^{n+1}, \zeta^{n+1}\right\rangle+\left\langle\left[\delta_{b_{1}^{n+1}, v^{n+1}, x}-\delta_{b_{1}^{n}, V^{n}, x}\right] v^{n+1}, \zeta^{n+1}\right\rangle\right\} \\
& -2 \Delta t\left\{\left\langle\delta_{b_{2}^{n}, V^{n}, y} \zeta^{n+1}, \zeta^{n+1}\right\rangle+\left\langle\left[\delta_{b_{2}^{n+1}, v^{n+1}, y}-\delta_{b_{2}^{n}, V^{n}, y}\right] v^{n+1}, \zeta^{n+1}\right\rangle\right\} \\
& -2 \Delta t\left\{\left\langle\delta_{b_{3}^{n}, V^{n}, z} \zeta^{n+1}, \zeta^{n+1}\right\rangle+\left\langle\left[\delta_{b_{3}^{n+1}, v^{n+1}, z}-\delta_{b_{3}^{n}, V^{n}, z}\right] v^{n+1}, \zeta^{n+1}\right\rangle\right\} \\
& -2(\Delta t)^{2}\left\{\left\langle\left(1+\frac{h}{2} \frac{\left|b_{1}^{n}\right|}{K_{3}\left(V^{n}\right)}\right)^{-1}\right.\right. \\
& \times \delta_{x}\left(K_{3}\left(V^{n}\right) \delta_{\bar{x}}\left(\hat{\Phi}_{3}^{-1}\left(1+\frac{h}{2} \frac{\left|b_{2}^{n}\right|}{K_{3}\left(V^{n}\right)}\right)^{-1} \delta_{y}\left(K_{3}\left(V^{n}\right) \delta_{\bar{y}} \zeta^{n+1}\right) \cdot\right), \zeta^{n+1}\right\rangle \\
& +\left\langle( 1 + \frac { h } { 2 } \frac { | b _ { 1 } ^ { n } | } { K _ { 3 } ( V ^ { n } ) } ) ^ { - 1 } \delta _ { x } \left(K _ { 3 } ( V ^ { n } ) \delta _ { \overline { x } } \left(\hat{\Phi}_{3}^{-1}\left(1+\frac{h}{2} \frac{\left|b_{2}^{n}\right|}{K_{3}\left(V^{n}\right)}\right)^{-1}\right.\right.\right. \\
& \left.\left.\times \delta_{y}\left(\left[K_{3}\left(v^{n+1}\right)-K_{3}\left(V^{n}\right)\right] \delta_{\bar{y}} v^{n+1}\right) \cdot\right), \zeta^{n+1}\right\rangle+\cdots \\
& +\left\langle[ ( 1 + \frac { h } { 2 } \frac { | b _ { 1 } ^ { n + 1 } | } { K _ { 3 } ( v ^ { n + 1 } ) } ) ^ { - 1 } - ( 1 + \frac { h } { 2 } \frac { | b _ { 1 } ^ { n } | } { K _ { 3 } ( V ^ { n } ) } ) ^ { - 1 } ] \delta _ { x } \left( K_{3}\left(v^{n+1}\right)\right.\right. \\
& \left.\left.\times \delta_{\bar{x}}\left(\hat{\Phi}_{1}^{-1}\left(1+\frac{h}{2} \frac{\left|b_{2}^{n+1}\right|}{K_{3}\left(v^{n+1}\right)}\right)^{-1} \delta_{y}\left(K_{3}\left(v^{n+1}\right) \delta_{\bar{y}} v^{n+1}\right) \cdot\right), \zeta^{n+1}\right\rangle\right\} \\
& -2(\Delta t)^{2}\left\{\left\langle( 1 + \frac { h } { 2 } \frac { | b _ { 1 } ^ { n } | } { K _ { 1 } ( V ^ { n } ) } ) ^ { - 1 } \delta _ { x } \left(K _ { 3 } ( V ^ { n } ) \delta _ { \overline { x } } \left(\hat{\Phi}_{1}^{-1}\left(1+\frac{h}{2} \frac{\left|b_{3}^{n}\right|}{K_{3}\left(V^{n}\right)}\right)^{-1}\right.\right.\right.\right. \\
& \left.\left.\left.\times \delta_{z}\left(K_{3}\left(V^{n}\right) \delta_{\bar{z}} \zeta^{n+1}\right) \cdot\right), \zeta^{n+1}\right\rangle+\cdots\right\}-2(\Delta t)^{2}\left\{\left\langle\left(1+\frac{h}{2} \frac{\left|b_{2}^{n}\right|}{K_{3}\left(V^{n}\right)}\right)^{-1}\right.\right.
\end{aligned}
$$




$$
\begin{aligned}
& \left.\times \delta_{y}\left(K_{3}\left(V^{n}\right) \delta_{\bar{y}}\left(\hat{\Phi}_{3}^{-1}\left(1+\frac{h}{2} \frac{\left|b_{3}^{n}\right|}{K_{3}\left(V^{n}\right)}\right)^{-1} \delta_{z}\left(K_{3}\left(V^{n}\right) \delta_{\bar{z}} \zeta^{n+1}\right) \cdot\right), \zeta^{n+1}\right\rangle+\cdots\right\} \\
& +2(\Delta t)^{2}\left\{\left\langle\delta_{b_{1}^{n}, V^{n}, x}\left(\hat{\Phi}_{3}^{-1}\left(1+\frac{h}{2} \frac{\left|b_{2}^{n}\right|}{K_{3}\left(V^{n}\right)}\right)^{-1} \delta_{y}\left(K_{3}\left(V^{n}\right) \delta_{\bar{y}} \zeta^{n+1}\right)\right), \zeta^{n+1}\right\rangle\right. \\
& +\left\langle\delta_{b_{1}^{n}, V^{n}, x}\left(\hat{\Phi}_{1}^{-1}\left(1+\frac{h}{2} \frac{\left|a_{2}^{n}\right|}{K_{3}\left(V^{n}\right)}\right)^{-1} \delta_{y}\left(\left[K_{3}\left(v^{n+1}\right)-K_{3}\left(V^{n}\right)\right] \delta_{\bar{y}} v^{n+1}\right)\right), \zeta^{n+1}\right\rangle \\
& +\cdots+\left\langle[ \delta _ { b _ { 1 } ^ { n + 1 } , v ^ { n + 1 } , x } - \delta _ { b _ { 1 } ^ { n } , V ^ { n } , x } ] \left(\hat{\Phi}_{3}^{-1}\left(1+\frac{h}{2} \frac{\left|b_{2}^{n+1}\right|}{K_{3}\left(v^{n+1}\right)}\right)^{-1}\right.\right. \\
& \left.\left.\left.\times \delta_{y}\left(K_{3}\left(v^{n+1}\right) \delta_{\bar{y}} v^{n+1}\right)\right), \zeta^{n+1}\right\rangle+\cdots\right\} \\
& -2(\Delta t)^{2}\left\{\left\langle\delta_{b_{1}^{n}, V^{n}, x}\left(\hat{\Phi}_{3}^{-1} \delta_{b_{2}^{n}, V^{n}, y} \zeta^{n+1}\right), \zeta^{n+1}\right\rangle\right. \\
& +\left\langle\delta_{b_{1}^{n}, V^{n}, x}\left(\hat{\Phi}_{3}^{-1}\left[\delta_{b_{2}^{n+1}, v^{n+1}, y}-\delta_{b_{2}^{n}, V^{n}, y}\right] v^{n+1}\right), \zeta^{n+1}\right\rangle \\
& \left.+\left\langle\left[\delta_{b_{1}^{n+1}, v^{n+1}, x}-\delta_{b_{1}^{n}, V^{n}, x}\right]\left(\hat{\Phi}_{1}^{-1} \delta_{b_{2}^{n+1}, v^{n+1}, y} v^{n+1}\right), \zeta^{n+1}\right\rangle+\cdots\right\} \\
& +2(\Delta t)^{3}\left\{\left\langle( 1 + \frac { h } { 2 } \frac { | b _ { 1 } ^ { n } | } { K _ { 1 } ( V ^ { n } ) } ) ^ { - 1 } \delta _ { x } \left(K _ { 3 } ( V ^ { n } ) \delta _ { \overline { x } } \left(\hat{\Phi}_{3}^{-1}\left(1+\frac{h}{2} \frac{\left|b_{2}^{n}\right|}{K_{3}\left(V^{n}\right)}\right)^{-1}\right.\right.\right.\right. \\
& \left.\times \delta_{y}\left(K_{3}\left(V^{n}\right) \delta_{\bar{y}}\left(\hat{\Phi}_{3}^{-1}\left(1+\frac{h}{2} \frac{\left|b_{3}^{n}\right|}{K_{3}\left(V^{n}\right)}\right)^{-1} \delta_{z}\left(K_{3}\left(V^{n}\right) \delta_{\bar{z}} \zeta^{n+1}\right) \cdot\right), \zeta^{n+1}\right\rangle+\cdots\right\} \\
& =2 \Delta t \sum_{i, j=1}^{N-1} K_{2, i j, \frac{1}{2}}^{n} \delta_{z} \omega_{i j, 0}^{n+1} \zeta_{i j, N}^{n+1} h^{2} \\
& +2 \Delta t\left\langle Q_{3}\left(v^{n+1}\right)-Q_{3}\left(V^{n}\right), \zeta^{n+1}\right\rangle+2 \Delta t\left\langle\varepsilon_{3}^{n+1}, \zeta^{n+1}\right\rangle . \\
& \left\{\left\|\Phi_{2}^{1 / 2} \omega^{n+1}\right\|^{2}-\left\|\Phi_{2}^{1 / 2} \omega^{n}\right\|^{2}\right\}+(\Delta t)^{2}\left\|\Phi_{2}^{1 / 2} d_{t} \omega^{n}\right\|^{2} \\
& =2 \Delta t\left\langle\delta_{z}\left(K_{2}^{n} \delta_{\bar{z}} \omega^{n+1}\right), \omega^{n+1}\right\rangle+2 \Delta t\left\langle\varepsilon_{2}^{n+1}, \omega^{n+1}\right\rangle \\
& =2 \Delta t \sum_{i, j, k=1}^{N-1} \delta_{z}\left(K_{2}^{n} \delta_{\bar{z}} \omega_{i j k}^{n+1}\right) \omega_{i j k}^{n+1} h^{3}+2 \Delta t\left\langle\varepsilon_{2}^{n+1}, \omega^{n+1}\right\rangle \\
& =2 \Delta t \sum_{i, j=1}^{N-1} \sum_{k=1}^{N-1} \omega_{i j k}^{n+1} \delta_{z}\left(K_{2}^{n} \delta_{\bar{z}} \omega^{n+1}\right) h^{3}+2 \Delta t\left\langle\varepsilon_{2}^{n+1}, \omega^{n+1}\right\rangle \\
& =-2 \Delta t \sum_{i, j=1}^{N-1} h^{2}\left\{\sum_{K=1}^{N} K_{2, i j k}^{n} \delta_{\bar{z}} \omega_{i j k}^{n+1} \cdot \delta_{\bar{z}} \omega_{i j k}^{n+1} h-\omega_{i j, N}^{n+1} K_{2, i j, N-\frac{1}{2}}^{n} \delta_{\bar{z}} \omega_{i j, N}^{n+1}\right. \\
& \left.+\omega_{i j, 0}^{n+1} K_{2, i j, \frac{1}{2}}^{n} \delta_{z} \omega_{i j, 0}^{n+1}\right\}+2 \Delta t\left\langle\varepsilon_{2}^{n+1}, \omega^{n+1}\right\rangle \\
& =-2 \Delta t\left(K_{2}^{n} \delta_{\bar{z}} \omega^{n+1}, \delta_{\bar{z}} \omega^{n+1}\right)+2 \Delta t \sum_{i, j=1}^{N-1}\left\{K_{2, i j, N-\frac{1}{2}}^{n} \delta_{\bar{z}} \omega_{i j, N}^{n+1} \cdot \xi_{i j, 0}^{n+1}\right. \\
& \left.-K_{2, i j, 1 / 2}^{n} \delta_{z} \omega_{i j, 0}^{n+1} \cdot \zeta_{i j, N}^{n+1}\right\} h^{2}+2 \Delta t\left\langle\varepsilon_{2}^{n+1}, \omega^{n+1}\right\rangle,
\end{aligned}
$$

此式经整理后可得

$$
\left\{\left\|\Phi_{2}^{1 / 2} \omega^{n+1}\right\|^{2}-\left\|\Phi_{2}^{1 / 2} \omega^{n}\right\|^{2}\right\}+(\Delta t)^{2}\left\|\Phi_{2}^{1 / 2} d_{t} \omega^{n}\right\|^{2}+2 \Delta t\left\|K_{2}^{1 / 2} \delta_{\bar{z}} \omega^{n+1}\right\|^{2}
$$




$$
\begin{aligned}
= & 2 \Delta t \sum_{i, j=1}^{N-1}\left\{K_{2, i j, N-\frac{1}{2}}^{n} \delta_{\bar{z}} \omega_{i j, N}^{n+1} \cdot \xi_{i j, 0}^{n+1}-K_{2, i j, 1 / 2}^{n} \delta_{z} \omega_{i j, 0}^{n+1} \cdot \zeta_{i j, N}^{n+1}\right\} h^{2} \\
& +2 \Delta t\left\langle\varepsilon_{2}^{n+1}, \omega^{n+1}\right\rangle .
\end{aligned}
$$

我们提出归纳法假定

$$
\sup _{0 \leqslant n \leqslant L} \max \left\{\left\|U^{n}\right\|_{1, \infty},\left\|V^{n}\right\|_{1, \infty}\right\} \leqslant M .
$$

现在估计 (16) 式左端第 3 项, 因为 $K_{1}$ 是正定的, 当 $h$ 适当小时, 有

$$
\begin{aligned}
& 2 \Delta t\left\{\left\langle K_{1}\left(U^{n}\right) \delta_{\bar{x}} \xi^{n+1}, \delta_{\bar{x}}\left(\left(1+\frac{h}{2} \frac{\left|a_{1}^{n}\right|}{K_{1}(U)}\right)^{-1} \xi^{n+1}\right)\right\rangle\right. \\
& +\left\langle\left[K_{1}\left(u^{n+1}\right)-K_{1}\left(U^{n}\right)\right] \delta_{\bar{x}} u^{n+1}, \delta_{\bar{x}}\left(\left(1+\frac{h}{2} \frac{\left|a_{1}^{n}\right|}{K_{1}(U)}\right)^{-1} \xi^{n+1}\right)\right\rangle \\
& +\left\langle\left[\left(1+\frac{h}{2} \frac{\left|a_{1}^{n+1}\right|}{K_{1}\left(u^{n+1}\right)}\right)^{-1}-\left(1+\frac{h}{2} \frac{\left|a_{1}^{n}\right|}{K_{1}\left(U^{n}\right)}\right)^{-1}\right]\right. \\
& \left.\left.+\delta_{x}\left(K_{1}\left(u^{n+1}\right) \delta_{\bar{x}} u^{n+1}\right), \xi^{n+1}\right\rangle\right\} \\
& =2 \Delta t\left\{\left\langle K_{1}\left(U^{n}\right) \delta_{\bar{x}} \xi^{n+1},\left(1+\frac{h}{2} \frac{\left|a_{1}^{n}\right|}{K_{1}\left(U^{n}\right)}\right)^{-1} \delta_{\bar{x}} \xi^{n+1}\right\rangle\right. \\
& +\left\langle K_{1}\left(U^{n}\right) \delta_{\bar{x}} \xi^{n+1}, \delta_{\bar{x}}\left(1+\frac{h}{2} \frac{\left|a_{1}^{n}\right|}{K_{1}\left(U^{n}\right)}\right)^{-1} \cdot \xi^{n+1}\right\rangle \\
& +\left\langle\left[K_{1}\left(u^{n+1}\right)-K_{1}\left(U^{n}\right)\right] \delta_{\bar{x}} u^{n+1},\left(1+\frac{h}{2} \frac{\left|a_{1}^{n}\right|}{K_{1}\left(U^{n}\right)}\right)^{-1} \delta_{\bar{x}} \xi^{n+1}\right. \\
& \left.+\delta_{\bar{x}}\left(1+\frac{h}{2} \frac{\left|a_{1}^{n}\right|}{K_{1}\left(U^{n}\right)}\right)^{-1} \cdot \xi^{n+1}\right\rangle \\
& +\left\langle\left[\left(1+\frac{h}{2} \frac{\left|a_{1}^{n+1}\right|}{K_{1}\left(u^{n+1}\right)}\right)^{-1}-\left(1+\frac{h}{2} \frac{\left|a_{1}^{n}\right|}{K_{1}\left(U^{n}\right)}\right)^{-1}\right]\right. \\
& \left.\left.+\delta_{x}\left(K_{1}\left(u^{n+1}\right) \delta_{\bar{x}} u^{n+1}\right), \xi^{n+1}\right\rangle\right\},
\end{aligned}
$$

于是可得

$$
\begin{aligned}
& 2 \Delta t\left\{\left\langle K_{1}\left(U^{n}\right) \delta_{\bar{x}} \xi^{n+1}, \delta_{\bar{x}}\left(\left(1+\frac{h}{2} \frac{\left|a_{1}^{n}\right|}{K_{1}\left(U^{n}\right)}\right)^{-1} \xi^{n+1}\right)\right\rangle\right. \\
& \left.+\left\langle\left[K_{1}\left(u^{n+1}\right)-K_{1}\left(U^{n}\right)\right] \delta_{\bar{x}} u^{n+1}, \delta_{\bar{x}}\left(\left(1+\frac{h}{2} \frac{\left|a_{1}^{n}\right|}{K_{1}\left(U^{n}\right)}\right)^{-1} \xi^{n+1}\right)\right\rangle+\cdots\right\} \\
\geqslant & \Delta t\left\|K_{1}^{n, 1 / 2} \delta_{\bar{x}} \xi^{n+1}\right\|^{2}-M\left\{\left\|\xi^{n+1}\right\|^{2}+\left\|\xi^{n}\right\|^{2}+(\Delta t)^{2}\right\} \Delta t,
\end{aligned}
$$

此处 $K_{1}^{n, 1 / 2}=K_{1}^{1 / 2}\left(U^{n}\right)$. 类似地对 (16) 式左端第 4 和 5 项, 同样有

$$
\begin{aligned}
& 2 \Delta t\left\{\left\langle K_{1}\left(U^{n}\right) \delta_{\bar{y}} \xi^{n+1}, \delta_{\bar{y}}\left(\left(1+\frac{h}{2} \frac{\left|a_{2}^{n}\right|}{K_{1}\left(U^{n}\right)}\right)^{-1} \xi^{n+1}\right)\right\rangle\right. \\
& +\left\langle\left[K_{1}\left(u^{n+1}\right)-K_{1}\left(U^{n}\right)\right] \delta_{\bar{y}} u^{n+1}, \delta_{\bar{y}}\left(\left(1+\frac{h}{2} \frac{\left|a_{2}^{n}\right|}{K_{1}\left(U^{n}\right)}\right)^{-1} \xi^{n+1}\right)\right\rangle \\
& +\left\langle\left[\left(1+\frac{h}{2} \frac{\left|a_{1}^{n+1}\right|}{K_{1}\left(u^{n+1}\right)}\right)^{-1}-\left(1+\frac{h}{2} \frac{\left|a_{1}^{n}\right|}{K_{1}\left(U^{n}\right)}\right)^{-1}\right]\right.
\end{aligned}
$$




$$
\begin{aligned}
& \left.\left.\times \delta_{y}\left(K_{1}\left(u^{n+1}\right) \delta_{\bar{y}} u^{n+1}\right), \xi^{n+1}\right\rangle\right\} \\
\geqslant & \Delta t\left\|K_{1}^{n, 1 / 2} \delta_{\bar{y}} \xi^{n+1}\right\|^{2}-M\left\{\left\|\xi^{n+1}\right\|^{2}+\left\|\xi^{n}\right\|^{2}+(\Delta t)^{2}\right\} \Delta t, \\
& 2 \Delta t\left\{\left\langle K_{1}\left(U^{n}\right) \delta_{\bar{z}} \xi^{n+1}, \delta_{\bar{z}}\left(\left(1+\frac{h}{2} \frac{\left|a_{3}^{n}\right|}{K_{1}\left(U^{n}\right)}\right)^{-1} \xi^{n+1}\right)\right\rangle\right. \\
& +\left\langle\left[K_{1}\left(u^{n+1}\right)-K_{1}\left(U^{n}\right)\right] \delta_{\bar{z}} u^{n+1}, \delta_{\bar{z}}\left(\left(1+\frac{h}{2} \frac{\left|a_{3}^{n}\right|}{K_{1}\left(U^{n}\right)}\right)^{-1} \xi^{n+1}\right)\right\rangle \\
& +\left\langle\left[\left(1+\frac{h}{2} \frac{\left|a_{3}^{n+1}\right|}{K_{1}\left(u^{n+1}\right)}\right)^{-1}-\left(1+\frac{h}{2} \frac{\left|a_{3}^{n}\right|}{K_{1}\left(U^{n}\right)}\right)^{-1}\right]\right. \\
& \left.\left.\times \delta_{z}\left(K_{1}\left(u^{n+1}\right) \delta_{\bar{z}} u^{n+1}\right), \xi^{n+1}\right\rangle\right\} \\
\geqslant & \Delta t\left\|K_{1}^{n, 1 / 2} \delta_{\bar{z}} \xi^{n+1}\right\|^{2}-M\left\{\left\|\xi^{n+1}\right\|^{2}+\left\|\xi^{n}\right\|^{2}+(\Delta t)^{2}\right\} \Delta t .
\end{aligned}
$$

下面估计 (16) 式右端诸项，第 1 项有下述估计：

$$
\begin{aligned}
& -2 \Delta t\left\{\left\langle\delta_{a_{1}^{n,} U^{n}, x} \xi^{n+1}, \xi^{n+1}\right\rangle+\left\langle\left[\delta_{a_{1}^{n+1}, u^{n+1}, x}-\delta_{a_{1}^{n}, U^{n}, x}\right] u^{n+1}, \xi^{n+1}\right\rangle\right\} \\
\leqslant & \varepsilon \sum_{i=1}^{N} \sum_{j, k=1}^{N=1}\left(\delta_{\bar{x}} \xi_{i j k}^{n+1}\right)^{2} h^{3} \Delta t+M\left\{\sum_{i, j, k=1}^{N-1}\left[\left(\xi_{i j k}^{n+1}\right)^{2}+\left(\xi_{i j k}^{n}\right)^{2}\right]^{2} h^{2}+(\Delta t)^{2}\right\} \Delta t \\
= & \varepsilon\left\|\delta_{\bar{x}} \xi^{n+1}\right\|^{2} \Delta t+M\left\{\left\|\xi^{n+1}\right\|^{2}+\left\|\xi^{n}\right\|^{2}+(\Delta t)^{2}\right\} \Delta t .
\end{aligned}
$$

类似地对第 2 和 3 项有

$$
\begin{aligned}
& -2 \Delta t\left\{\left\langle\delta_{a_{2}^{n}, V^{n}, y} \xi^{n+1}, \xi^{n+1}\right\rangle+\left\langle\left[\delta_{a_{2}^{n+1}, u^{n+1}, y}-\delta_{a_{2}^{n}, V^{n}, y}\right] u^{n+1}, \xi^{n+1}\right\rangle\right\} \\
\leqslant & \varepsilon\left\|\delta_{\bar{y}} \xi^{n+1}\right\|^{2} \Delta t+M\left\{\left\|\xi^{n+1}\right\|^{2}+\left\|\xi^{n}\right\|^{2}+(\Delta t)^{2}\right\} \Delta t, \\
& -2 \Delta t\left\{\left\langle\delta_{a_{3}^{n}, U^{n}, z} \xi^{n+1}, \xi^{n+1}\right\rangle+\left\langle\left[\delta_{a_{3}^{n+1}, u^{n+1}, z}-\delta_{a_{3}^{n}, U^{n}, z}\right] u^{n+1}, \xi^{n+1}\right\rangle\right\} \\
\leqslant & \varepsilon\left\|\delta_{\bar{z}} \xi^{n+1}\right\|^{2} \Delta t+M\left\{\left\|\xi^{n+1}\right\|^{2}+\left\|\xi^{n}\right\|^{2}+(\Delta t)^{2}\right\} \Delta t .
\end{aligned}
$$

现重点讨论第 4 项

$$
\begin{aligned}
& -2(\Delta t)^{2}\left\{\left\langle\left(1+\frac{h}{2} \frac{\left|a_{1}^{n}\right|}{K_{1}\left(U^{n}\right)}\right)^{-1} \delta_{x}\left(K_{1}\left(U^{n}\right) \delta_{\bar{x}}\left(\hat{\Phi}_{1}^{-1} \delta_{y}\left(K_{1}\left(U^{n}\right) \delta_{\bar{y}} \xi^{n+1}\right) \cdot\right), \xi^{n+1}\right\rangle\right.\right. \\
& +\left\langle( 1 + \frac { h } { 2 } \frac { | a _ { 1 } ^ { n } | } { K _ { 1 } ( U ^ { n } ) } ) ^ { - 1 } \delta _ { x } \left(K _ { 1 } ( U ^ { n } ) \delta _ { \overline { x } } \left(\hat{\Phi}_{1}^{-1}\left(1+\frac{h}{2} \frac{\left|a_{2}^{n}\right|}{K_{1}\left(U^{n}\right)}\right)^{-1}\right.\right.\right. \\
& \left.\left.\times \delta_{y}\left(\left[K_{1}\left(u^{n+1}\right)-K_{1}\left(U^{n}\right)\right] \delta_{\bar{y}} u^{n+1}\right) \cdot\right), \xi^{n+1}\right\rangle+\cdots \\
& +\left\langle\left[\left(1+\frac{h}{2} \frac{\left|a_{1}^{n+1}\right|}{K_{1}\left(u^{n+1}\right)}\right)^{-1}-\left(1+\frac{h}{2} \frac{\left|a_{1}^{n}\right|}{K_{1}\left(U^{n}\right)}\right)^{-1}\right]\right. \\
& \left.\times \delta_{x}\left(K_{1}\left(u^{n+1}\right) \delta_{\bar{x}}\left(\Phi_{1}^{-1}\left(1+\frac{h}{2} \frac{\left|a_{2}^{n+1}\right|}{K_{1}\left(u^{n+1}\right)}\right)^{-1} \delta_{y}\left(K_{1}\left(u^{n+1}\right) \delta_{\bar{y}} u^{n+1}\right) \cdot\right), \xi^{n+1}\right\rangle\right\} .
\end{aligned}
$$

首先讨论其首项，尽管 $-\delta_{x}\left(K_{1}\left(U^{n}\right) \delta_{\bar{x}}\right),-\delta_{y}\left(K_{1}\left(U^{n}\right) \delta_{\bar{y}}\right), \cdots$ 是自共轭的正定算 子, 空间区域为正方形, 但它们的乘积一般是不可交换的. 记

$$
r_{a_{1}}^{n}=\left(1+\frac{\left|a_{1}^{n}\right|}{K_{1}\left(U^{n}\right)}\right)^{-1}, \quad r_{a_{2}}^{n}=\left(1+\frac{h}{2} \frac{\left|a_{2}^{n}\right|}{K_{1}\left(U^{n}\right)}\right)^{-1},
$$


利用

$$
\delta_{x} \delta_{y}=\delta_{y} \delta_{x}, \quad \delta_{x} \delta_{\bar{y}}=\delta_{\bar{y}} \delta_{x}, \quad \delta_{\bar{x}} \delta_{y}=\delta_{y} \delta_{\bar{x}}, \quad \cdots
$$

有

$$
\begin{aligned}
& -2(\Delta t)^{2}\left\langle r_{a_{1}}^{n} \delta_{x}\left(K_{1}\left(U^{n}\right) \delta_{\bar{x}}\left(\hat{\Phi}_{1}^{-1} r_{a_{1}}^{n} \delta_{y}\left(K_{1}\left(U^{n}\right) \delta_{\bar{y}} \xi^{n+1}\right) \cdot\right), \xi^{n+1}\right\rangle\right. \\
& =-2(\Delta t)^{2}\left\{\left\langleK_{1}\left(U^{n}\right) \delta_{\bar{x}} \delta_{\bar{y}} \xi^{n+1}+\delta_{\bar{x}} K_{1}\left(U^{n}\right) \cdot \delta_{\bar{y}} \xi^{n+1}, \hat{\Phi}_{1}^{-1} r_{a_{2}}^{n} K_{1}\left(U^{n}\right) \delta_{\bar{x}} \delta_{\bar{y}} \xi^{n+1}\right.\right. \\
& \left.+\hat{\Phi}_{1}^{-1} r_{a_{2}}^{n} K_{1}\left(U^{n}\right) \delta_{\bar{x}} \delta_{\bar{y}} r_{a_{1}}^{n} \cdot \xi^{n+1}+\delta_{\bar{y}}\left(\hat{\Phi}_{1}^{-1} r_{a_{2}}^{n} K_{1}\left(U^{n}\right)\right) \cdot \delta_{\bar{x}}\left(r_{a_{1}}^{n} \xi^{n+1}\right)\right\rangle \\
& +\left\langle K_{1}\left(U^{n}\right) \delta_{\bar{y}} \xi^{n+1}, \delta_{\bar{x}}\left(\hat{\Phi}_{1}^{-1} r_{a_{2}}^{n}\right) \cdot K_{1}\left(U^{n}\right) \delta_{\bar{x}} \delta_{\bar{y}}\left(r_{a_{1}}^{n} \xi^{n+1}\right)\right. \\
& \left.\left.+\delta_{\bar{y}}\left(\delta_{\bar{x}}\left(\hat{\Phi}_{1}^{-1} r_{a_{2}}^{n}\right) \cdot K_{1}\left(U^{n}\right)\right) \cdot \delta_{\bar{x}}\left(r_{a_{1}}^{n} \xi^{n+1}\right)\right\rangle\right\} \\
& =-2(\Delta t)^{2} \sum_{i, j, k=1}^{N}\left\{K_{1}\left(U^{n}\right)_{i, j-\frac{1}{2}, k} \cdot K_{1}\left(U^{n}\right)_{i-\frac{1}{2}, j k} \hat{\Phi}_{1, i j k}^{-1} r_{a_{2}, i j k}^{n} r_{a_{1}, i j k}^{n}\left[\delta_{\bar{x}} \delta_{\bar{y}} \xi_{i j k}^{n+1}\right]^{2}\right. \\
& +\left[K_{1}\left(U^{n}\right)_{i, j-\frac{1}{2}, k} \delta_{\bar{y}}\left(\hat{\Phi}_{1, i j k}^{-1} r_{a_{2}, i j k}^{n} K_{1}\left(U^{n}\right)_{i-\frac{1}{2}, j k}\right) \cdot \delta_{\bar{x}}\left(r_{a_{1}, i j k}^{n} \xi_{i j k}^{n+1}\right)\right. \\
& +\hat{\Phi}_{1, i j k}^{-1} r_{a_{2}, i j k}^{n} K_{1}\left(U^{n}\right)_{i-\frac{1}{2}, j k} \delta_{\bar{x}} K_{1}\left(U^{n}\right)_{i-\frac{1}{2}, j k} \delta_{\bar{y}} \xi_{i j k}^{n+1} \\
& +K_{1}\left(U^{n}\right)_{i, j-\frac{1}{2}, k} \cdot K_{1}\left(U^{n}\right)_{i-\frac{1}{2}, j k} \delta_{\bar{x}}\left(\hat{\Phi}_{1, i j k}^{-1} \cdot r_{a_{2}, i j k}^{n}\right) r_{a_{1}, i j k}^{n} \cdot \delta_{\bar{y}} \xi_{i j k}^{n+1} \\
& \left.+K_{1}\left(U^{n}\right)_{i, j-\frac{1}{2}, k} \cdot K_{1}\left(U^{n}\right)_{i, j-\frac{1}{2}, k} \hat{\Phi}_{1, i j k}^{-1} \cdot r_{a_{2}, i j k}^{n} \cdot \delta_{\bar{x}} \delta_{\bar{y}} r_{a_{2}, i j k}^{n} \cdot \xi_{i j k}^{n+1}\right] \\
& \times \delta_{\bar{x}} \delta_{\bar{y}} \xi_{i j k}^{n+1}+\left[\delta_{\bar{x}} K_{1}\left(U^{n}\right)_{i, j-\frac{1}{2}, k} \cdot \delta_{\bar{y}}\left(\hat{\Phi}_{1, i j k}^{-1} \cdot r_{a_{2}, i j k}^{n} K_{1}\left(U^{n}\right)_{i-\frac{1}{2}, j k}\right) r_{a, i j k}^{n}\right. \\
& \left.+K_{1}\left(U^{n}\right)_{i, j-\frac{1}{2}, k} \delta_{\bar{y}}\left(\delta_{\bar{x}}\left(\hat{\Phi}_{1, i j k}^{-1} r_{a_{2}, i j k}^{n}\right) \cdot K_{1}\left(U^{n}\right)_{i-\frac{1}{2}, j k}\right) r_{a_{1}, i j k}^{n}\right] \\
& \times \delta_{\bar{x}} \xi_{i j k}^{n+1} \cdot \delta_{\bar{y}} \xi_{i j k}^{n+1}+\delta_{\bar{x}} K_{1}\left(U^{n}\right)_{i, j-\frac{1}{2}, k}\left[\hat{\Phi}_{1, i j k}^{-1} \cdot r_{a_{2}, i j k}^{n} K_{1}\left(U^{n}\right)_{i-\frac{1}{2}, j k} \delta_{\bar{x}} \delta_{\bar{y}} r_{a_{1}, i j k}^{n}\right. \\
& \left.+\delta_{\bar{y}}\left(\hat{\Phi}_{1, i j k}^{-1} r_{a_{2}, i j k}^{n} K_{1}\left(U^{n}\right)_{i-\frac{1}{2}, j k}\right) \delta_{\bar{x}} r_{a_{1}, i j k}^{n}\right] \\
& +K_{1}\left(U^{n}\right)_{i, j-\frac{1}{2}, k} \cdot K_{1}\left(U^{n}\right)_{i-\frac{1}{2}, j k} \delta_{\bar{x}}\left(\hat{\Phi}_{1, i j k}^{-1} r_{a_{2}, i j k}^{n}\right) \delta_{\bar{x}} \delta_{\bar{y}} r_{a_{1}, i j k}^{n} \\
& +K_{1}\left(U^{n}\right)_{i, j-\frac{1}{2}, k} \delta_{\bar{y}}\left(\delta_{\bar{x}}\left(\hat{\Phi}_{1, i j k}^{-1} r_{a_{2}, i j k}^{n}\right) \cdot K_{1}\left(U^{n}\right)_{i, j-\frac{1}{2}, k}\right) \\
& \left.\times \delta_{\bar{x}} r_{a, i j k}^{n} \xi_{i j k}^{n+1} \delta_{\bar{y}} \xi_{i j k}^{n+1}\right\} h^{3} .
\end{aligned}
$$

对上述表达式的前 2 项, 应用 $K_{1}, \hat{\Phi}_{1}^{-1}, r_{a_{1}}^{n}, r_{a_{2}}^{n}$ 的正定性和归纳法假定 (19) 式 可分离出高阶差商项 $\delta_{\bar{x}} \delta_{\bar{y}} \xi_{i j k}^{n+1}$, 再利用 Cauchy 不等式可得

$$
\begin{aligned}
& -2(\Delta t)^{2} \sum_{i, j, k=1}^{N}\left\{K_{1}\left(U^{n}\right)_{i, j-\frac{1}{2}, k} \cdot K_{1}\left(U^{n}\right)_{i-\frac{1}{2}, j k} \hat{\Phi}_{1, i j k}^{-1} r_{a_{2}, i j k}^{n} r_{a_{1}, i j k}^{n}\left[\delta_{\bar{x}} \delta_{\bar{y}} \xi_{i j k}^{n+1}\right]^{2}\right. \\
& +\left[K_{1}\left(U^{n}\right)_{i, j-\frac{1}{2}, k} \delta_{\bar{y}}\left(\hat{\Phi}_{1, i j k}^{n} r_{a_{2}, i j k}^{n} \cdot K_{1}\left(U^{n}\right)_{i-\frac{1}{2}, j k}\right) \cdot \delta_{\bar{x}}\left(r_{a_{1}, i j k}^{n} \xi_{i j k}^{n+1}\right)\right. \\
& +\hat{\Phi}_{1, i j k}^{n} r_{a_{2}, i j k}^{n} \cdot K_{1}\left(U^{n}\right)_{i-\frac{1}{2}, j k} \cdot \delta_{\bar{x}} K_{1}\left(U^{n}\right)_{i, j-\frac{1}{2}, k} \cdot \delta_{\bar{y}} \xi_{i j k}^{n+1} \\
& +K_{1}\left(U^{n}\right)_{i, j-\frac{1}{2}, k} K_{1}\left(U^{n}\right)_{i-\frac{1}{2}, j k} \delta_{\bar{x}}\left(\hat{\Phi}_{1, i j k}^{-1} \cdot r_{a_{2}, i j k}^{n}\right) r_{a_{1}, i j k}^{n} \cdot \delta_{\bar{y}} \xi_{i j k}^{n+1} \\
& \left.+K_{1}\left(U^{n}\right)_{i, j-\frac{1}{2}, k} K_{1}\left(U^{n}\right)_{i-\frac{1}{2}, j k} \hat{\Phi}_{1, i j k}^{-1} \cdot r_{a_{2}, i j k}^{n} \cdot \delta_{\bar{x}} \delta_{\bar{y}} r_{a_{1}, i j k}^{n} \cdot \xi_{i j k}^{n+1}\right] \delta_{\bar{x}} \delta_{\bar{y}} \xi_{i j k}^{n+1} h^{2} \\
& \leqslant-\left(K_{*}\right)^{2}\left(\Phi^{*}\right)^{-1}(\Delta t)^{2} \sum_{i, j, k=1}^{N}\left[\delta_{\bar{x}} \delta_{\bar{y}} \xi^{n+1}\right]^{2} h^{3}
\end{aligned}
$$




$$
\begin{aligned}
& +M(\Delta t)^{2}\left\{\left\|\delta_{\bar{x}} \xi^{n+1}\right\|^{2}+\left\|\delta_{\bar{y}} \xi^{n+1}\right\|^{2}+\left\|\xi^{n+1}\right\|^{2}\right\}, \\
& -2(\Delta t)^{2} \sum_{i, j, k=1}^{N}\left\{\left[\delta_{\bar{x}} K_{1}\left(U^{n}\right)_{i, j-\frac{1}{2}, k} \cdot \delta_{\bar{y}}\left(\hat{\Phi}_{1, i j k}^{-1} r_{a_{2}, i j k}^{n} K_{1}\left(U^{n}\right)_{i-\frac{1}{2}, j k}\right) r_{a_{1}, i j k}^{n}\right.\right. \\
& \left.+K_{1}\left(U^{n}\right)_{i, j-\frac{1}{2}, k} \delta_{\bar{y}}\left(\delta_{\bar{x}}\left(\hat{\Phi}_{1, i j k}^{-1} r_{a_{2}, i j k}^{n}\right) \cdot K_{1}\left(U^{n}\right)_{i-\frac{1}{2}, j k}\right) r_{a_{1}, i j k}^{n}\right] \delta_{\bar{x}} \xi_{i j k}^{n+1} \delta_{\bar{y}} \xi_{i j k}^{n+1} \\
& +\left[\delta _ { \overline { x } } K _ { 1 } ( U ^ { n } ) _ { i , j - \frac { 1 } { 2 } , k } \left(\hat{\Phi}_{1, i j k}^{-1} r_{a_{2}, i j k}^{n} K_{1}\left(U^{n}\right)_{i-\frac{1}{2}, j k} \delta_{\bar{x}} \delta_{\bar{y}} r_{a_{1}, i j k}^{n}\right.\right. \\
& \left.+\delta_{\bar{y}}\left(\hat{\Phi}_{1, i j k}^{-1} r_{a_{2}, i j k}^{n} K_{1}\left(U^{n}\right)_{i-\frac{1}{2}, j k}\right) \delta_{\bar{x}} r_{a_{1}, i j k}^{n}\right) \\
& +K_{1}\left(U^{n}\right)_{i, j-\frac{1}{2}, k} \cdot K_{1}\left(U^{n}\right)_{i-\frac{1}{2}, j k} \delta_{\bar{x}}\left(\hat{\Phi}_{1, i j k}^{-1} r_{a_{1}, i j k}^{n}\right) \delta_{\bar{x}} \delta_{\bar{y}} r_{a_{1}, i j k}^{n} \\
& \left.\left.+K_{1}\left(U^{n}\right)_{i, j-\frac{1}{2}, k} \delta_{\bar{y}}\left(\delta_{\bar{x}} \hat{\Phi}_{1, i j k}^{-1} r_{a_{2}, i j k}^{n}\right) \cdot K_{1}\left(U^{n}\right)_{i-\frac{1}{2}, j k} \cdot \delta_{\bar{x}} r_{a_{2}, i j k}^{n}\right] \xi_{i j k}^{n+1} \delta_{\bar{y}} \xi_{i j k}^{n+1}\right\} h^{3} \\
& \leqslant M(\Delta t)^{2}\left\{\left\|\delta_{\bar{x}} \xi^{n+1}\right\|^{2}+\left\|\delta_{\bar{y}} \xi^{n+1}\right\|^{2}+\left\|\xi^{n+1}\right\|^{2}\right\} \text {. } \\
& \text { 对其余诸项, 经类似的分析有 } \\
& -2(\Delta t)^{2}\left\{\left\langle( 1 + \frac { h } { 2 } \frac { | a _ { 1 } ^ { n } | } { K _ { 1 } ( U ^ { n } ) } ) ^ { - 1 } \delta _ { x } \left(K _ { 1 } ( U ^ { n } ) \delta _ { \overline { x } } \left(\hat{\Phi}_{1}^{-1}\left(1+\frac{h}{2} \frac{\left|a_{2}^{n}\right|}{K_{1}\left(U^{n}\right)}\right)^{-1}\right.\right.\right.\right. \\
& \left.\left.\times \delta_{y}\left(\left[K_{1}\left(u^{n+1}\right)-K_{1}\left(U^{n}\right)\right] \delta_{\bar{y}} u^{n+1}\right) \cdot\right), \xi^{n+1}\right\rangle+\cdots \\
& +\left\langle\left[\left(1+\frac{h}{2} \frac{\left|a_{1}^{n+1}\right|}{K_{1}\left(u^{n+1}\right)}\right)^{-1}-\left(1+\frac{h}{2} \frac{\left|a_{1}^{n}\right|}{K_{1}\left(U^{n}\right)}\right)^{-1}\right]\right. \\
& \left.\left.\times \delta_{x}\left(K_{1}\left(u^{n+1}\right) \delta_{\bar{x}}\left(\hat{\Phi}_{1}^{-1}\left(1+\frac{h}{2} \frac{\left|a_{2}^{n+1}\right|}{K_{1}\left(u^{n+1}\right)}\right)^{-1} \delta_{y}\left(u^{n+1}\right) \delta_{\bar{y}} u^{n+1}\right)\right), \xi^{n+1}\right\rangle\right\} \\
& \leqslant \varepsilon(\Delta t)^{2} \sum_{i, j, k=1}^{N}\left[\delta_{\bar{x}} \delta_{\bar{y}} \xi_{i j k}^{n+1}\right]^{2} h^{3}+M(\Delta t)^{2}\left\{\left\|\delta_{\bar{x}} \xi^{n+1}\right\|^{2}+\left\|\delta_{\bar{y}} \xi^{n+1}\right\|^{2}+\left\|\xi^{n+1}\right\|^{2}+\left\|\xi^{n}\right\|^{2}\right\} .
\end{aligned}
$$

综合上述诸估计式, 对第 4 项有

$$
\begin{aligned}
&- 2(\Delta t)^{2}\left\{\left\langle\left(1+\frac{h}{2} \frac{\left|a_{1}^{n}\right|}{K_{1}\left(U^{n}\right)}\right)^{-1} \delta_{x}\left(K_{1}\left(U^{n}\right) \delta_{\bar{x}}\left(\hat{\Phi}_{1}^{-1} \delta_{y}\left(K_{1}\left(U^{n}\right) \delta_{\bar{y}} \xi^{n+1}\right) \cdot\right), \xi^{n+1}\right\rangle\right.\right. \\
&+ \cdots+\left\langle\left[\left(1+\frac{h}{2} \frac{\left|a_{1}^{n+1}\right|}{K_{1}\left(u^{n+1}\right)}\right)^{-1}-\left(1+\frac{h}{2} \frac{\left|a_{1}^{n}\right|}{K_{1}\left(U^{n}\right)}\right)^{-1}\right]\right. \\
& \times\left.\delta_{x}\left(K_{1}\left(u^{n+1}\right) \delta_{\bar{x}}\left(\hat{\Phi}_{1}^{-1}\left(1+\frac{h}{2} \frac{\left|a_{2}^{n+1}\right|}{K_{1}\left(u^{n+1}\right)}\right)^{-1} \delta_{y}\left(K_{1}\left(u^{n+1}\right) \delta_{\bar{y}} u^{n+1}\right) \cdot\right), \xi^{n+1}\right\rangle\right\} \\
& \leqslant-\frac{1}{2}\left(K_{*}\right)^{2}\left(\Phi^{*}\right)^{-1}(\Delta t)^{2} \sum_{i, j, k=1}^{N}\left[\delta_{\bar{x}} \delta_{\bar{y}} \xi_{i j k}^{n+1}\right]^{2} h^{3} \\
&+ M\left\{\left\|\delta_{\bar{x}} \xi^{n+1}\right\|^{2}+\left\|\delta_{\bar{y}} \xi^{n+1}\right\|^{2}+\left\|\xi^{n+1}\right\|^{2}+\left\|\xi^{n}\right\|^{2}\right\} \Delta t . \\
& \quad \text { 对第 } 5 \text { 和 } 6 \text { 项的估计是类似的. 对第 } 7 \text { 项可得估计式 } \\
& \quad 2(\Delta t)^{2}\left\{\left\langle\left(1+\frac{h}{2} \frac{\left|a_{1}^{n}\right|}{K_{1}\left(U^{n}\right)}\right)^{-1} \delta_{x}\left(K_{1}\left(U^{n}\right) \delta_{\bar{x}}\left(\hat{\Phi}_{1}^{-1}\left[\delta_{a_{2}^{n}, U^{n}, y} \xi^{n+1}\right) \cdot\right), \xi^{n+1}\right\rangle\right.\right. \\
& \quad+\cdots+\left\langle\left[\left(1+\frac{h}{2} \frac{\left|a_{1}^{n+1}\right|}{K_{1}\left(u^{n+1}\right)}\right)^{-1}-\left(1+\frac{h}{2} \frac{\left|a_{1}^{n}\right|}{K_{1}\left(U^{n}\right)}\right)^{-1}\right]\right. \\
&\left.\quad \times \delta_{x}\left(K_{1}\left(u^{n+1}\right) \delta_{\bar{x}}\left(\hat{\Phi}_{1}^{-1}\left(\delta_{a_{2}^{n+1}, u^{n+1}, y} u^{n+1}\right) \cdot\right), \xi^{n+1}\right\rangle\right\}
\end{aligned}
$$




$$
\begin{aligned}
\leqslant & \varepsilon(\Delta t)^{2} \sum_{i, j, k=1}^{N}\left[\delta_{\bar{x}} \delta_{\bar{y}} \xi_{i j k}^{n+1}\right]^{2} h^{3} \\
& +M(\Delta t)^{2}\left\{\left\|\delta_{\bar{x}} \xi^{n+1}\right\|^{2}+\left\|\delta_{\bar{y}} \xi^{n+1}\right\|^{2}+\left\|\xi^{n+1}\right\|^{2}+\left\|\xi^{n}\right\|^{2}\right\} .
\end{aligned}
$$

对第 8 和 9 项同样可得类似的估计式:

$$
\begin{aligned}
& 2(\Delta t)^{2}\left\{\left\langle\delta_{a_{1}^{n}, U^{n}, x}\left(\hat{\Phi}_{1}^{-1}\left(1+\frac{h}{2} \frac{\left|a_{2}^{n}\right|}{K_{1}\left(U^{n}\right)}\right)^{-1} \delta_{y}\left(K_{1}\left(U^{n}\right) \delta_{\bar{y}} \xi^{n+1}\right)\right), \xi^{n+1}\right\rangle+\cdots\right\} \\
& -2(\Delta t)^{2}\left\{\left\langle\delta_{a_{1}^{n} U^{n}, x}\left(\hat{\Phi}_{1}^{-1} \delta_{a_{2}^{n}, U^{n}, y} \xi^{n+1}\right), \xi^{n+1}\right\rangle+\cdots\right\} \\
\leqslant & \varepsilon(\Delta t)^{2} \sum_{i, j, k=1}^{N}\left[\delta_{\bar{x}} \delta_{\bar{y}} \xi_{i j k}^{n+1}\right]^{2} h^{3} \\
& +M(\Delta t)^{2}\left\{\left\|\delta_{\bar{x}} \xi^{n+1}\right\|^{2}+\left\|\delta_{\bar{y}} \xi^{n+1}\right\|^{2}+\left\|\xi^{n+1}\right\|^{2}+\left\|\xi^{n}\right\|^{2}\right\} .
\end{aligned}
$$

对 (16) 式右端第 10 项, 类似地估计可得

$$
\begin{aligned}
& 2(\Delta t)^{3}\left\{\left\langle( 1 + \frac { h } { 2 } \frac { | a _ { 1 } ^ { n } | } { K _ { 1 } ( U ^ { n } ) } ) ^ { - 1 } \delta _ { x } \left(K _ { 1 } ( U ^ { n } ) \delta _ { \overline { x } } \left(\hat{\Phi}_{1}^{-1}\left(1+\frac{h}{2} \frac{\left|a_{2}^{n}\right|}{K_{1}\left(U^{n}\right)}\right)^{-1}\right.\right.\right.\right. \\
& \left.\times \delta_{y}\left(K_{1}\left(U^{n}\right) \delta_{\bar{y}}\left(\hat{\Phi}_{1}^{-1}\left(1+\frac{h}{2} \frac{\left|a_{3}^{n}\right|}{K_{1}\left(U^{n}\right)}\right)^{-1} \delta_{z}\left(K_{1}\left(U^{n}\right) \delta_{\bar{z}} \xi^{n+1}\right) \cdot\right), \xi^{n+1}\right\rangle+\cdots\right\} \\
\leqslant & M(\Delta t)^{2}\left\{\left\|\delta_{\bar{x}} \xi^{n+1}\right\|^{2}+\left\|\delta_{\bar{y}} \xi^{n+1}\right\|^{2}+\left\|\delta_{\bar{z}} \xi^{n+1}\right\|^{2}+\left\|\xi^{n+1}\right\|^{2}+\left\|\xi^{n}\right\|^{2}\right\} .
\end{aligned}
$$

对 (16) 式右端最后两项, 由 $\varepsilon_{0}$-Lipschitz 条件可得

$$
\begin{aligned}
& 2 \Delta t\left\langle Q_{1}\left(u^{n+1}\right)-Q_{1}\left(U^{n+1}\right), \xi^{n+1}\right\rangle+2 \Delta t\left\langle\varepsilon_{1}^{n+1}, \xi^{n+1}\right\rangle \\
\leqslant & M \Delta t\left\{\left\|\xi^{n+1}\right\|^{2}+(\Delta t)^{2}\right\} .
\end{aligned}
$$

对误差方程 (16), 利用 (20) 式经整理可得

$$
\begin{aligned}
& \left\|\hat{\Phi}_{2}^{1 / 2} \xi^{n+1}\right\|^{2}-\left\|\hat{\Phi}_{1}^{1 / 2} \xi^{n}\right\|^{2}+(\Delta t)^{2}\left\|\hat{\Phi}_{2}^{1 / 2} d_{t} \xi^{n}\right\|^{2} \\
& +\Delta t\left\{\left\|K_{1}^{n, 1 / 2} \delta_{\bar{x}} \xi^{n+1}\right\|^{2}+\left\|K_{1}^{n, 1 / 2} \delta_{\bar{y}} \xi^{n+1}\right\|^{2}+\left\|K_{1}^{n, 1 / 2} \delta_{\bar{z}} \xi^{n+1}\right\|^{2}\right\} \\
\leqslant & M\left\{(\Delta t)^{2}+h^{4}+\left\|\xi^{n+1}\right\|^{2}+\left\|\xi^{n}\right\|^{2}\right\} \Delta t \\
& -2 \Delta t \sum_{i, j=1}^{N-1} K_{2, i j, N-\frac{1}{2}}{ }^{n} \delta_{\bar{z}} \omega_{i j, N}^{n+1} \xi_{i j, 0}^{n+1} h^{2} .
\end{aligned}
$$

对误差方程 (17) 可进行类似的估计, 经整理可得

$$
\begin{aligned}
& \left\|\hat{\Phi}_{2}^{1 / 2} \zeta^{n+1}\right\|^{2}-\left\|\hat{\Phi}_{1}^{1 / 2} \zeta^{n}\right\|^{2}+(\Delta t)^{2}\left\|\hat{\Phi}_{2}^{1 / 2} d_{t} \zeta^{n}\right\|^{2} \\
& +\Delta t\left\{\left\|K_{3}^{n, 1 / 2} \delta_{\bar{x}} \zeta^{n+1}\right\|^{2}+\left\|K_{3}^{n, 1 / 2} \delta_{\bar{y}} \zeta^{n+1}\right\|^{2}+\left\|K_{3}^{n, 1 / 2} \delta_{\bar{z}} \zeta^{n+1}\right\|^{2}\right\} \\
\leqslant & M\left\{(\Delta t)^{2}+h^{4}+\left\|\zeta^{n+1}\right\|^{2}+\left\|\zeta^{n}\right\|^{2}\right\} \Delta t \\
& +2 \Delta t \sum_{i, j=1}^{N-1} K_{2, i j, 1 / 2}^{n} \delta_{z} \omega_{i j, 0}^{n+1} \zeta_{i j, N}^{n+1} h^{2}
\end{aligned}
$$


此时对误差方程 (18) 有

$$
\begin{aligned}
& \left\|\Phi_{2}^{1 / 2} \omega^{n+1}\right\|^{2}-\left\|\Phi_{2}^{1 / 2} \omega^{n}\right\|^{2}+(\Delta t)^{2}\left\|\Phi_{2}^{1 / 2} d_{t} \omega^{n}\right\|^{2}+2 \Delta t\left\|K_{2}^{1 / 2} \delta_{\bar{z}} \omega^{n+1}\right\|^{2} \\
\leqslant & 2 \Delta t \sum_{i, j=1}^{N-1}\left\{K_{2, i j, N-\frac{1}{2}}^{n} \delta_{\bar{z}} \omega_{i j, N}^{n+1} \cdot \xi_{i j, 0}^{n+1}-K_{2, i j, 1 / 2}^{n} \delta_{z} \omega_{i j, 0}^{n+1} \cdot \zeta_{i j, N}^{n+1}\right\} h^{2} \\
& +M \Delta t\left\{(\Delta t)^{2}+h^{4}+\left\|\omega^{n+1}\right\|^{2}\right\} .
\end{aligned}
$$

联合 (21) (23) 式, 并对时间 $t$ 求和 $(0 \leqslant n \leqslant L)$, 注意到 $\xi^{0}=\xi^{0}=\omega^{0}=0$, 故有

$$
\begin{aligned}
& \left\{\left\|\hat{\Phi}_{1}^{1 / 2} \xi^{L+1}\right\|^{2}+\left\|\hat{\Phi}_{3}^{1 / 2} \xi^{L+1}\right\|^{2}+\left\|\Phi_{2}^{1 / 2} \omega^{L+1}\right\|^{2}\right\} \\
& +\Delta t \sum_{n=0}^{L}\left\{\left\|\hat{\Phi}_{1}^{1 / 2} d_{t} \xi^{n}\right\|^{2}+\left\|\hat{\Phi}_{3}^{1 / 2} d_{t} \zeta^{n}\right\|^{2}+\left\|\Phi_{2}^{1 / 2} d_{t} \omega^{n}\right\|^{2}\right\} \Delta t \\
& \quad+\sum_{n=0}^{L}\left\{\left\|K_{1}^{n, 1 / 2} \delta_{\bar{x}} \xi^{n+1}\right\|^{2}+\left\|K_{1}^{n, 1 / 2} \delta_{\bar{y}} \xi^{n+1}\right\|^{2}+\left\|K_{1}^{n, 1 / 2} \delta_{\bar{z}} \xi^{n+1}\right\|^{2}\right. \\
& \left.\quad+\left\|K_{3}^{n, 1 / 2} \delta_{\bar{x}} \zeta^{n+1}\right\|^{2}+\left\|K_{3}^{n, 1 / 2} \delta_{\bar{y}} \zeta^{n+1}\right\|+\left\|K_{3}^{n, 1 / 2} \delta_{\bar{z}} \zeta^{n+1}\right\|^{2}+\left\|\Phi_{2}^{1 / 2} \delta_{\bar{z}} \omega^{n+1}\right\|^{2}\right\} \Delta t \\
& \leqslant M\left\{\sum_{n=0}^{L}\left[\left\|\xi^{n+1}\right\|^{2}+\left\|\zeta^{n+1}\right\|^{2}+\left\|\omega^{n+1}\right\|^{2}\right] \Delta t+(\Delta t)^{2}+h^{4}\right\} .
\end{aligned}
$$

应用 Gronwall 引理可得

$$
\begin{aligned}
& \left\{\left\|\hat{\Phi}_{1}^{1 / 2} \xi^{L+1}\right\|^{2}+\left\|\hat{\Phi}_{3}^{1 / 2} \zeta^{L+1}\right\|^{2}+\left\|\Phi_{2}^{1 / 2} \omega^{L+1}\right\|^{2}\right\} \\
& +\Delta t \sum_{n=0}^{L}\left\{\left\|\hat{\Phi}_{1}^{1 / 2} d_{t} \xi^{n}\right\|^{2}+\left\|\hat{\Phi}_{3}^{1 / 2} d_{t} \zeta^{n}\right\|^{2}+\left\|\Phi_{2}^{1 / 2} d_{t} \omega^{n}\right\|^{2}\right\} \Delta t \\
& +\sum_{n=0}^{L}\left\{\left\|K_{1}^{n, 1 / 2} \delta_{\bar{x}} \xi^{n+1}\right\|^{2}+\left\|K_{1}^{n, 1 / 2} \delta_{\bar{y}} \xi^{n+1}\right\|^{2}+\left\|K_{1}^{n, 1 / 2} \delta_{\bar{z}} \xi^{n+1}\right\|^{2}\right. \\
& \left.+\left\|K_{3}^{n, 1 / 2} \delta_{\bar{x}} \zeta^{n+1}\right\|^{2}+\left\|K_{3}^{n, 1 / 2} \delta_{\bar{y}} \zeta^{n+1}\right\|+\left\|K_{3}^{n, 1 / 2} \delta_{\bar{z}} \zeta^{n+1}\right\|^{2}+\left\|K_{2}^{n, 1 / 2} \delta_{\bar{z}} \omega^{n+1}\right\|\right\} \Delta t \\
& \leqslant M\left\{(\Delta t)^{2}+h^{4}\right\} .
\end{aligned}
$$

最后需要检验归纳法假定 (19) 式. 因为

$$
\left\|U^{n}\right\|_{1, \infty} \leqslant\left\|\xi^{n}\right\|_{1, \infty}+\left\|u^{n}\right\|_{1, \infty}, \quad\left\|V^{n}\right\|_{1, \infty} \leqslant\left\|\zeta^{n}\right\|_{1, \infty}+\left\|v^{n}\right\|_{1, \infty},
$$

因此只要检验

$$
\sup _{0 \leqslant n \leqslant L} \max \left\{\left\|\xi^{n}\right\|_{1, \infty},\left\|\zeta^{n}\right\|_{1, \infty}\right\} \leqslant M .
$$

对 $n=0$, 我们有 $\xi^{0}=\zeta^{0}=0$, 归纳法假定 (19) 式显然是正确的. 若 $n=1,2, \cdots, L$ 时 (19) 式成立, 则当 $n=L+1$ 时, 由 (25) 式, 若 $\Delta t \leqslant M h^{2}$, 则有

$$
\begin{aligned}
\left\|\xi^{L+1}\right\|_{1, \infty}+\left\|\zeta^{L+1}\right\|_{1, \infty} & \leqslant M h^{-1}\left\{\left\|\xi^{L+1}\right\|_{1}+\left\|\zeta^{L+1}\right\|_{1}\right\} \\
& \leqslant M h^{-2}\left\{\left\|\xi^{L+1}\right\|_{0}+\left\|\zeta^{L+1}\right\|_{0}\right\} \\
& \leqslant M h^{-2}\left\{h^{2}+\Delta t\right\} \leqslant M,
\end{aligned}
$$

归纳法假定 (19) 式得证. 
定理 假定问题 (1) (4) 的精确解满足光滑性条件:

$$
\begin{gathered}
\frac{\partial^{2} u}{\partial t^{2}} \in L^{\infty}\left(L^{\infty}\left(\Omega_{1}\right)\right), \quad u \in L^{\infty}\left(W^{4, \infty}\left(\Omega_{1}\right)\right) \cap W^{1, \infty}\left(W^{1, \infty}\left(\Omega_{1}\right)\right), \\
\frac{\partial^{2} v}{\partial t^{2}} \in L^{\infty}\left(L^{\infty}\left(\Omega_{3}\right)\right), \quad v \in L^{\infty}\left(W^{4, \infty}\left(\Omega_{3}\right)\right) \cap W^{1, \infty}\left(W^{1, \infty}\left(\Omega_{3}\right)\right), \\
\frac{\partial^{2} w}{\partial t^{2}} \in L^{\infty}\left(L^{\infty}\left(\Omega_{2}\right)\right), \quad w \in L^{\infty}\left(W^{4, \infty}\left(\Omega_{2}\right)\right) .
\end{gathered}
$$

采用迎风分数步差分格式 (7)、(8) 和 (10) 逐层计算, 则下述误差估计式成立:

$$
\begin{aligned}
& \quad\|u-U\|_{\bar{L}^{\infty}\left(J ; l^{2}\right)}+\|v-V\|_{\bar{L}^{\infty}\left(J ; l^{2}\right)}+\|w-W\|_{\bar{L}^{\infty}\left(J ; l^{2}\right)} \\
& \quad+\|u-U\|_{\bar{L}^{2}\left(J ; h^{1}\right)}+\|v-V\|_{\bar{L}^{2}\left(J ; h^{1}\right)}+\|w-W\|_{\bar{L}^{2}\left(J ; h^{1}\right)} \\
& \leqslant M^{*}\left\{\Delta t+h^{2}\right\},
\end{aligned}
$$

此处

$$
\|g\|_{\bar{L}^{\infty}(J ; x)}=\sup _{n \Delta t \leqslant T}\left\|f^{n}\right\|_{x}, \quad\|g\|_{\bar{L}^{2}(J ; x)}=\sup _{L \Delta t \leqslant T}\left\{\sum_{n=0}^{L}\left\|g^{n}\right\|_{x}^{2} \Delta t\right\}^{1 / 2},
$$

$M^{*}$ 依赖于函数 $u, v, w$ 及其导数.

\section{4 实际应用}

\section{1 油资源运移聚集三维数值模拟}

本文所提出的方法已成功地应用于胜利油田东营凹陷的单层三维数值模拟, 问题的数学模型是下述一组非线性偏微分方程初边程值问题:

$$
\begin{gathered}
\nabla \cdot\left(K \frac{k r_{\mathrm{o}}}{\mu_{\mathrm{o}}} \nabla \psi_{\mathrm{o}}\right)+B_{\mathrm{o}} q=-\Phi s^{\prime}\left(\frac{\partial \psi_{\mathrm{o}}}{\partial t}-\frac{\partial \psi_{\mathrm{w}}}{\partial t}\right), \\
X=(x, y, z)^{\mathrm{T}} \in \Omega, \quad t \in J=(0, T], \\
\nabla \cdot\left(k \frac{k r_{\mathrm{w}}}{\mu_{\mathrm{w}}} \nabla \psi_{\mathrm{w}}\right)+B_{\mathrm{w}} q=\Phi s^{\prime}\left(\frac{\partial \psi_{\mathrm{o}}}{\partial t}-\frac{\partial \psi_{\mathrm{w}}}{\partial t}\right), \quad X \in \Omega, t \in J,
\end{gathered}
$$

此处 $\psi_{\mathrm{o}}$ 和 $\psi_{\mathrm{w}}$ 是油相和水相流动位势.

数值模拟结果表明 ${ }^{1)}$ :

i) 渗流力学三维数值模拟过程可再现油水在真实的三维空间中二次运移聚 集的演化全过程. 模拟结果符合油水运移聚集的渗流力学特征. 油聚集到一定 饱和度后, 在油水位势综合作用下, 油在三维空间中从高位势向低位势方向运 移，在油的局部低位势区，发生聚集，并可能成藏; 时间越长，排烃量越大，聚 集的油越多，最后聚集到油位势低的区域，形成油藏.

ii) 渗流力学数值模拟结果与东营凹陷的实际油田分布情形对比，模拟结果 成藏位置与实际纯化等油田的位置基本吻合，在储油强度方面也与实际地质资 源情形基本一致.

1) 参见 1399 页脚注 1 
iii) 数千万年的超长时间的成功数值模拟表明我们的数值模拟方法是强稳 定的、高效率的、高精度的.

\section{2 油资源运移聚集多层数值模拟}

对于多层问题的油资源运移聚集数值模拟，应用本方法对滩海地区亦已有 初步成果, 问题的数学模型是

$$
\begin{gathered}
\nabla \cdot\left(K_{1} \frac{k r_{\mathrm{o}}}{\mu_{\mathrm{o}}} \nabla \psi_{\mathrm{o}}\right)+B_{\mathrm{o}} q-\left(K_{2} \frac{k r_{\mathrm{o}}}{\mu_{\mathrm{o}}} \frac{\partial \psi}{\partial z}\right)_{z=H_{2}} \\
=-\Phi s^{\prime}\left(\frac{\partial \psi_{\mathrm{o}}}{\partial t}-\frac{\partial \psi_{\mathrm{w}}}{\partial t}\right), \quad X \in \Omega_{1}, t \in J \\
\nabla \cdot\left(K_{1} \frac{k r_{\mathrm{w}}}{\mu_{\mathrm{w}}} \nabla \psi_{W}\right)+B_{\mathrm{w}} q-\left(K_{2} \frac{k r_{\mathrm{w}}}{\mu_{\mathrm{w}}} \frac{\partial \psi}{\partial z}\right)_{z=H_{2}} \\
=\Phi s^{\prime}\left(\frac{\partial \psi_{\mathrm{o}}}{\partial t}-\frac{\partial \psi_{\mathrm{w}}}{\partial t}\right), \quad X \in \Omega_{1}, t \in J, \\
\frac{\partial}{\partial z}\left(K_{2} \frac{k r_{\mathrm{o}}}{\mu_{\mathrm{o}}} \frac{\partial \psi_{\mathrm{o}}}{\partial z}\right)=-\Phi s^{\prime}\left(\frac{\partial \psi_{\mathrm{o}}}{\partial t}-\frac{\partial \psi_{\mathrm{w}}}{\partial t}\right), \quad X \in \Omega_{2}, t \in J \\
\frac{\partial}{\partial z}\left(K_{2} \frac{k r_{\mathrm{w}}}{\mu_{\mathrm{w}}} \frac{\partial \psi_{\mathrm{w}}}{\partial z}\right)=\Phi s^{\prime}\left(\frac{\partial \psi_{\mathrm{o}}}{\partial t}-\frac{\partial \psi_{\mathrm{w}}}{\partial t}\right), \quad X \in \Omega_{2}, t \in J \\
\nabla \cdot\left(K_{3} \frac{k r_{\mathrm{o}}}{\mu_{\mathrm{o}}} \nabla \psi_{\mathrm{o}}\right)+B_{\mathrm{w}} q+\left(K_{2} \frac{k r_{\mathrm{o}}}{\mu_{\mathrm{o}}} \frac{\partial \psi_{\mathrm{o}}}{\partial z}\right)_{z=H_{1}} \\
=-\Phi s^{\prime}\left(\frac{\partial \psi_{\mathrm{o}}}{\partial t}-\frac{\partial \psi_{\mathrm{w}}}{\partial t}\right), \quad X \in \Omega_{3}, t \in J \\
\nabla \cdot\left(K_{3} \frac{k r_{\mathrm{w}}}{\mu_{\mathrm{w}}} \nabla \psi_{\mathrm{w}}\right)+B_{\mathrm{w}} q+\left(K_{3} \frac{k r_{\mathrm{w}}}{\mu_{\mathrm{w}}} \frac{\partial \psi_{\mathrm{o}}}{\partial z}\right)_{z=H_{1}} \\
=\Phi s^{\prime}\left(\frac{\partial \psi_{\mathrm{o}}}{\partial t}-\frac{\partial \psi_{\mathrm{w}}}{\partial t}\right), \quad X \in \Omega_{3}, t \in J
\end{gathered}
$$

此处 $\psi_{\mathrm{o}}$ 和 $\psi_{\mathrm{w}}$ 是油相和水相流动位势.

数值模拟结果表明 ${ }^{1)}$ :

从计算结果看出沙三下段和沙三中段的油沿着断层向沙三上段运移, 并在 洼陷周围的隆起和斜坡带聚集，即埕岛地区、老河口、五号桩及孤东地区，这与 胜利油田勘探形势的现状基本吻合.

致谢 本课题的研究曾与 R. E. Ewing 和姜礼尚教授讨论过, 作者在此表示 感谢.

\section{参考文献}

1 Ewing R E. The Mathematics of Reservoir Simulation. Philadelphia: SIAM, 1983

2 Bredehoeft J D, Pinder G F. Digital analysis of areal flow in multiaquifer groundwater systems: a quasithree-dimensional model. Water Resources Research, 1970, 6(3): 888 893

1) 参见 1399 页脚注 2 
3 Don W, Emil O F. An iterative quasi-three-dimensional finite element model for heterogeneous multiaquifer systems. Water Resources Research, 1978, 14(5): 943 952

4 Ungerer P. Fluid flow, hydrocarbon generation and migration. AAPG Bull, 1990, 74(3): 309 335

5 Douglas Jr J, Russell T F. Numerical method for convection-dominated diffusion problems based on combining the method of characteristics with finite element or finite difference procedures. SIAM J Numer Anal, 1982, 19(5): 871 885

6 Douglas Jr J. Finite difference methods for two-phase incompressible flow in porous media. SIAM J Numer Anal, 1983, 20(4): 681 696

7 Peaceman D W. Fundamental of Numerical Reservoir Simulation. Amsterdam: Elsevier, 1980

8 Marchuk G I. Splitting and alternating direction method. In: Ciarlet P G, Lions J L, eds. Handbook of Numerical Analysis. Paris: Elesevior Science Publishers, 1990. 197 460

9 Ewing R E, Lazarov R D, Vassilev A T. Finite difference scheme for parabolic problems on a composite grids with refinement in time and space. SIAM J Numer Anal, 1994, 31(6): 1605 1622

10 Lazarov R D, Mischev I D, Vassilevski P S. Finite volume methods for convection-diffusion problems. SIAM J Numer Anal, 1996, 33(1): 31 55

11 袁益让. 可压缩两相驱动问题的分数步长特征差分格式. 中国科学, A 辑, 1998, 28(10): 893 902

12 袁益让. 多层渗流方程组合系统的迎风分数步差分方法. 中国科学, A 辑, 2001, 31(9): 791 806

13 萨马尔斯基 A A, 安德烈耶夫 B B. 椭圆型方程差分方法. 北京: 科学出版社, 1994

14 袁益让. 油藏数值模拟中动边值问题的特征差分方法. 中国科学, A 辑, 1994, 24(10): 1029 1036

15 袁益让. 强化采油数值模拟的特征差分方法和 $l^{2}$ 估计. 中国科学, A 辑, 1993, 23(8): 801 810 